\title{
A Combination of G Protein-Coupled Receptor Modulators Protects Photoreceptors from Degeneration ${ }^{[\mathrm{S}}$
}

\author{
Tivadar Orban, ${ }^{1}$ Henri Leinonen, ${ }^{1}$ Tamar Getter, Zhiqian Dong, Wenyu Sun, Songqi Gao, \\ Alexander Veenstra, Hossein Heidari-Torkabadi, Timothy S. Kern, Philip D. Kiser, \\ and Krzysztof Palczewski
}

Department of Pharmacology, Cleveland Center for Membrane and Structural Biology, School of Medicine, Case Western Reserve University, Cleveland, Ohio (T.O., H.L., T.G., S.G., A.V., H.H.-T., T.S.K., P.D.K., K.P.); Research Service, Louis Stokes Cleveland Veterans Affairs Medical Center, Cleveland, Ohio (T.S.K., P.D.K.); and Polgenix Inc., Cleveland, Ohio (Z.D., W.S.)

Received September 16, 2017; accepted November 20, 2017

\begin{abstract}
Degeneration of retinal photoreceptor cells can arise from environmental and/or genetic causes. Since photoreceptor cells, the retinal pigment epithelium (RPE), neurons, and glial cells of the retina are intimately associated, all cell types eventually are affected by retinal degenerative diseases. Such diseases often originate either in rod and/or cone photoreceptor cells or the RPE. Of these, cone cells located in the central retina are especially important for daily human activity. Here we describe the protection of cone cells by a combination therapy consisting of the $G$ protein-coupled receptor modulators metoprolol, tamsulosin, and bromocriptine. These drugs were tested in $\mathrm{Abca}^{-1-} \mathrm{Rdh8^{-1- }}$ mice, a preclinical model for retinal degeneration. The specificity of these drugs was determined with an essentially complete panel of human G protein-coupled receptors. Significantly, the combination of metoprolol, tamsulosin, and bromocriptine had no
\end{abstract}

deleterious effects on electroretinographic responses of wildtype mice. Moreover, putative $\mathrm{G}$ protein-coupled receptor targets of these drugs were shown to be expressed in human and mouse eyes by RNA sequencing and quantitative polymerase chain reaction. Liquid chromatography together with mass spectrometry using validated internal standards confirmed that metoprolol, tamsulosin, and bromocriptine individually or together penetrate the eye after either intraperitoneal delivery or oral gavage. Collectively, these findings support human trials with combined therapy composed of lower doses of metoprolol, tamsulosin, and bromocriptine designed to safely impede retinal degeneration associated with certain genetic diseases (e.g., Stargardt disease). The same low-dose combination also could protect the retina against diseases with complex or unknown etiologies such as age-related macular degeneration.

\section{Introduction}

Retinal function depends on a monolayer of pigmented epithelial cells, densely packed rod and cone photoreceptors, and a downstream connection of neurons and glial cells (McBee et al., 2001). Of these, rod and cone cells are

This research was supported in part by the National Institutes of Health National Eye Institute [Core Grants P30EY011373 and P30EY025585 and Grants R24EY024864 (to T.S.K.) and R01EY009339, R24EY027283, and EY024864 (to K.P.)] and the Department of Veterans Affairs [Grant IK2BX002683 (to P.D.K.)]. K.P. is the John H. Hord Professor of Pharmacology. K.P. is an inventor of U.S. patent numbers 8722669 ("Compounds and Methods of Treating Ocular Disorders") and 20080275134 ("Methods for Treatment of Retinal Degenerative Disease") issued to Case Western Reserve University (CWRU). The value of these patents may be affected by this publication. CWRU may license this technology for commercial development. K.P. is the Chief Scientific Officer of Polgenix Inc.

${ }^{1}$ T.O. and H.L. contributed equally to this work.

https://doi.org/10.1124/jpet.117.245167.

S This article has supplemental material available at jpet.aspetjournals.org. asymmetrically distributed in the human retina with a rodenriched periphery and cone-dominated fovea (Curcio et al., 1990). Ultimately, photoreceptor depletion is the central pathologic manifestation underlying disparate retinal degenerative disorders, including Stargardt disease and age-related macular degeneration (Curcio et al., 1996; Wenzel et al., 2005).

Systems pharmacology comprises an approach that can identify drug combinations that serve as prophylactics against cell death (Chen and Palczewski, 2015), which is critical for neural tissues like the retina that lack regenerative properties. G protein-coupled receptors (GPCRs) constitute a large family of transmembrane receptors that regulate intracellular signaling essential for cellular homeostasis, playing critical roles in the physiology of virtually all biologic processes. They and their regulated pathways are targets for more than $30 \%$ of clinically used drugs (Katritch et al., 2013). We previously demonstrated through a systems pharmacology approach that

ABBREVIATIONS: AR, adrenergic receptor; BBB, blood-brain barrier; BLE, bright light exposure; BRM, bromocriptine; CWRU, Case Western Reserve University; DMSO, dimethylsulfoxide; DOX, doxazosin; DR, dopamine receptor; ERG, electroretinography; FPKM, fragments per kilobase of transcript per million mapped reads; GPCR, G protein-coupled receptor; ISI, interstimulus interval; LC, liquid chromatography; LED, light-emitting diode; MS, mass spectrometry; MTP, metoprolol; NIMH, National Institute of Mental Health; OCT, optical coherence tomography; ONH, optic nerve head; ONL, outer nuclear layer; PBS, phosphate-buffered saline; PBST, phosphate-buffered saline containing $0.5 \%$ Triton X-100; PCR, polymerase chain reaction; qPCR, quantitative polymerase chain reaction; RNA-seq, RNA sequencing; RPE, retinal pigment epithelium; TAM, tamsulosin; TEV, tobacco etch virus; tTA, tetracycline-controlled transcriptional activator. 
a combination of Food and Drug Administration-approved drugs targeting GPCRs protected retinas against bright light-induced loss of rod photoreceptor cells and the impairment of secondary neurons (Chen et al., 2016). These drugs included metoprolol (MTP), an antagonist of $\mathrm{G}_{\mathrm{s}}$-coupled $\beta_{1}$-adrenergic receptors (ARs); tamsulosin (TAM), an antagonist of $\mathrm{G}_{\mathrm{q}}$-coupled $\alpha_{1}$-ARs; doxazosin (DOX), an antagonist of $\mathrm{G}_{\mathrm{q}}$-coupled $\alpha_{1}$-ARs; and bromocriptine (BRM), an agonist for $\mathrm{G}_{\mathrm{i}}$-coupled D2 and D3 dopamine receptors (DRD2 and DRD3).

While significant advances have been made toward improved treatments for retinal degenerative diseases, critical questions must be addressed before advancing a systems pharmacology approach to human trials. First, similar to rods, can cone photoreceptor cell structure and function be preserved by pretreatment with GPCR modulators? Second, do proposed GPCR modulators used individually or in combination have specificities sufficient to ensure safe and effective treatment? This concern is especially important for compounds like BRM with a broad spectrum of action (Maj et al., 1977; Rosenfeld et al., 1980). Third, are endogenous GPCRs affected during bright light exposure (BLE) rescued by combinatorial treatment in an animal model of acute retinal degeneration? Fourth, is retinal function adversely affected in normal animals by GPCR modulators? Finally, are the pharmacokinetic and pharmacodynamic properties of these drugs appropriate for the treatment of human ocular diseases?

This study demonstrates that cone photoreceptor cells can be effectively protected by a low-dose combination treatment with GPCR modulators. These drugs, achieving effective levels in the eye, affected GPCR signaling in a mouse model of bright light-induced retinal degeneration without adversely affecting retinal function in wild-type mice. These findings demonstrate that drugs can be delivered in combination at decreased doses to ensure a therapeutic benefit with fewer side effects than with monotherapy.

\section{Materials and Methods}

Human Retinal Tissue. For RNA sequencing (RNA-seq) experiments, human retinal tissue was obtained from a patient at the Cleveland Clinic Cole Eye Institute (Cleveland, OH). The retina was carefully dissected from the patient's untreated eye that required enucleation owing to the occurrence of a large ocular melanoma, and the tissue was immediately placed in RNAlater (Qiagen, Valencia, CA). The retinal sample was obtained from a hemi-retina free of tumor. This eye had no abnormal neovascularization of the iris or retina and lacked signs of inflammation. Subsequent procedures were described in a prior publication (Mustafi et al., 2013a).

For polymerase chain reaction (PCR) experiments, retinal RNA was obtained from Dr. A. Maeda and Dr. B. Sahu [Case Western Reserve University (CWRU), Cleveland, $\mathrm{OH}$, originally derived from the donor eye of a female patient with diabetes who had died from breast cancer. Total RNA was reverse transcribed into cDNA with a highcapacity cDNA reverse transcription kit from Applied Biosystems (Foster City, CA), following the manufacturer's protocol. Final PCR products were analyzed on a $2 \%$ agarose gel to confirm their expected sizes. All experiments were performed in accordance with the provisions of the Declaration of Helsinki and were approved by the institutional review boards at CWRU. Donor eyes were obtained from Eversight Ohio (Cleveland, $\mathrm{OH}$ ).

Animals. All animal procedures and experiments conformed to the recommendations of the American Veterinary Medical Association Panel on Euthanasia and were approved by the Institutional Animal Care and Use Committee of CWRU and conducted in accordance with the Association for Research in Vision and Ophthalmology Statement for the Use of Animals in Ophthalmic and Visual Research. Mice were housed in the animal research center at CWRU School of Medicine, where they received a standard chow diet (LabDiet 5053; LabDiet, St. Louis, MO) and were kept in a 12-hour/12-hour light/dark cyclic environment.

Male and female C57BL/6J, albino B6(Cg)-Tyr $r^{c-2 J} / \mathrm{J}$, and BALB/cJ mice were obtained from the Jackson Laboratory (Bar Harbor, ME) and acclimated for at least 6 days after their arrival. Mice were used at 4-8 weeks of age. Male and female $A b c a 4^{-1-} R d h 8^{-1-}$ mice were genotyped by established methods (Maeda et al., 2009; Chen et al., 2012). Only Rd8 mutation-free mice with the Leu variation at amino

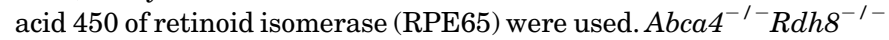
mice with a mixed background, $129 \mathrm{SV}$ or C57BL/, and their siblings were employed for most experiments. Pupils of pigmented mice were dilated with $1 \%$ tropicamide ophthalmic solution (Henry Schein Inc., Melville, NY) before BLE.

Drugs and Their Isotope Standards. MTP, TAM, DOX, and BRM for pharmacokinetic studies were obtained from Tocris Inc. (Avonmouth, Bristol, UK). MTP and TAM also were purchased from TCI America (Portland, OR) and BRM was from Enzo Life Sciences (Ann Arbor, MI) for cone protection experiments. Deuterated internal standards for MTP (MTP-d7), DOX (DOX-d8), and TAM (TAM-d5) (CDN Isotopes, Pointe-Claire, QC, Canada) and BRM ( $\alpha$-ergocryptine) (Sigma-Aldrich, St. Louis, MO) were solubilized with methanol and water [50:50 (v/v)] to a final concentration of $250 \mathrm{mg} / \mathrm{ml}$. Further dilutions were made with water and filtered through a $0.22-\mu \mathrm{m}$ pore membrane. Drug solutions and deuterated standards were kept at $-80^{\circ} \mathrm{C}$ and used within 3 months after solubilization. Stock solutions were checked monthly against freshly prepared standards of known concentrations. Solvents including formic acid, sterile dimethylsulfoxide (DMSO), and acetonitrile were purchased from Sigma-Aldrich, whereas methanol was from Fisher Scientific (Pittsburgh, PA).

Mice and Bright Light-Induced Retinopathy. $A b c a 4^{-/-} R d h 8^{-/-}$ mice, a model of photoreceptor degeneration (Maeda et al., 2008), were used for experiments assessing the efficacy of MTP, TAM, and BRM triple treatment against bright light-induced retinopathy. Twenty-four female mice and 17 male mice at 5-8 weeks of age were dark-adapted overnight before acute BLE. The next morning, mice received an intraperitoneal injection of drug solution (10 mg/kg MTP, $0.5 \mathrm{mg} / \mathrm{kg}$ TAM, and $1 \mathrm{mg} / \mathrm{kg}$ BRM; $150 \mu \mathrm{l}$ volume) or vehicle (2\% DMSO, $2 \%$ propylene glycol, and $96 \%$ saline; $150 \mu \mathrm{l}$ volume) and their pupils were dilated with $1 \%$ tropicamide eye drops. All animal handling was performed under dim red light. BLE was initiated $0.5,2$, or 4 hours after drug injections. Freely moving mice were exposed for 0.5 hour to bright white light from light-emitting diode (LED) flood lights (85-265 V, $100 \mathrm{~W}, 6500 \mathrm{~K}$ color temperature) set at two sides of their Plexiglas home cages. Luminance, measured at the center of the cage, was set at 25 klux (L203 Photometer; Macam Photometrics Ltd., Livingston, UK) after the light sensor was positioned upward. Sufficient pupil dilation was confirmed visually before each BLE experiment, and extra eye drops were added in experiments that were initiated 2 or 4 hours after drug or vehicle injections.

In Vivo Anatomic Imaging and Functional Assessment of Mouse Retina. Retinal anatomy and function were assessed in vivo with optical coherence tomography (OCT) and electroretinography (ERG) 7 days after BLE. Mice were anesthetized with Rodent Cocktail as specified by Institutional Animal Care and Use Committee [20 $\mathrm{mg} / \mathrm{ml}$ ketamine and $1.75 \mathrm{mg} / \mathrm{ml}$ xylazine in phosphate-buffered saline (PBS)] at a dose of $0.1-0.13 \mathrm{ml} / 25 \mathrm{~g}$ b.w. by intraperitoneal injection, and their pupils were dilated with $1 \%$ tropicamide. OCT imaging was performed with a Bioptigen spectral-domain OCT device (Leica Microsystems Inc., Buffalo Grove, IL). Four frames of OCT b-scan images were acquired from a series of 1200 a-scans. The thickness of the retinal outer nuclear layer (ONL) was measured $500 \mu \mathrm{m}$ away from the optic nerve head (ONH) in four retinal quadrants (i.e., nasal, temporal, superior, and inferior quadrants) using a ruler tool in the OCT imaging software. Mean ONL thickness 
of two eyes was averaged for each mouse and these averages were used for further statistical analyses.

ERG measurements were performed immediately after OCT imaging with a Diagnosys Celeris rodent ERG device (Diagnosys, Lowell, MA). A mouse was placed on a heating pad at $37.4^{\circ} \mathrm{C}$, and its pupils were moistened with $2.5 \%$ hypromellose eye lubricant (HUB Pharmaceuticals, Rancho Cucamonga, CA). Light stimulation was produced by an in-house scripted stimulation series in Espion software (version 6; Diagnosys). First, eyes were adapted to a white background light $[6500 \mathrm{~K}]$ at $20 \mathrm{~cd} / \mathrm{m}^{2}$ and then stimulated with a green LED [peak emission at $\sim 544 \mathrm{~nm}$, bandwidth $\sim 160 \mathrm{~nm}$ ] at light intensity increments of $0.1 \mathrm{~cd} \cdot \mathrm{s} / \mathrm{m}^{2}$ [interstimulus interval (ISI) 400 milliseconds, 50 repetitions averaged], 0.5 [ISI 400 milliseconds, 30 repetitions], 1.0 [ISI 500 milliseconds, 25 repetitions], 5.0 [ISI 1 second, 15 repetitions], and $10.0 \mathrm{~cd} \cdot \mathrm{s} / \mathrm{m}^{2}$ [ISI 2 seconds, 10 repetitions]. Then the background light was switched to a green light at $20 \mathrm{~cd} / \mathrm{m}^{2}$ and eyes were adapted for 1 minute before stimulation was performed with a blue LED (peak emission at $\sim 460 \mathrm{~nm}$, bandwidth $\sim 100 \mathrm{~nm}$ ). The stimulation series employed with the blue LED was similar to that with the green LED. As the mouse short wavelength cone opsin (Sopsin) and medium wavelength cone opsin (M-opsin) sensitivities peak at 360 and $508 \mathrm{~nm}$ (Nikonov et al., 2006), the green LED predominantly stimulates cones containing M-opsin, whereas the blue LED stimulates cones containing both S- and M-opsins. LED light emission spectra were measured with a Specbos 1211UV spectroradiometer (JETI Technische Instrumente GmbH, Jena, Germany). The ERG signal was acquired at $2 \mathrm{kHz}$ and filtered with a low frequency cutoff at $0.25 \mathrm{~Hz}$ and a high frequency cutoff at $300 \mathrm{~Hz}$. Espion software automatically detected the ERG a-waves (first negative ERG component) and b-waves (first positive ERG component). Averaged b-wave amplitudes from the two eyes, measured from the a-wave trough, were used in the statistical analyses.

Immunohistochemistry and Microscopy of Retinal Whole Mounts. At day 8 after BLE, mice were euthanized by cervical dislocation, the superior side of their eyes was marked, and their eyes were enucleated. Eyes were fixed in $4 \%$ paraformaldehyde in PBS composed of $1.7 \mathrm{mM} \mathrm{KHPO}_{4}, 5.2 \mathrm{mM} \mathrm{Na}_{2} \mathrm{HPO}_{4}$, and $0.15 \mathrm{M} \mathrm{NaCl}$ at $\mathrm{pH}$ 7.0-7.6 for 1 hour before dissection. The cornea was removed following the orientation of the ora serrata, and the lens and vitreous were removed from each eye cup. Retinas were dissected from the eyecups and further fixed and flattened in $4 \%$ paraformaldehyde between two coverslips for $0.5-1$ hour. After fixation, retinas were washed two times in phosphate-buffered saline containing $0.5 \%$ Triton $\mathrm{X}-100$ (PBST). Next, retinas floating in PBST were frozen at $-80^{\circ} \mathrm{C}$ for 10 minutes and then thawed at room temperature to increase tissue permeability. Thawed retinas were incubated for 3 nights at $4^{\circ} \mathrm{C}$ in goat polyclonal S-opsin antibody (catalog number sc-14363, 1:4000 dilution; Santa Cruz Biotechnology, Dallas, TX) and rabbit polyclonal M-opsin antibody (catalog number NB110-74730, 1:1000 dilution; Novus Biologicals, Littleton, CO) in PBST containing 5\% normal donkey serum. After incubation with primary antibodies, retinas were washed three times for 10 minutes in PBST and then incubated for 2 hours at room temperature with secondary antibodies (catalog number ab150129, Abcam donkey anti-goat Alexa Fluor 488, and catalog number ab150075, donkey anti-rabbit Alexa Fluor 647, at dilutions of 1:500 for both; Abcam, Cambridge, UK) in PBST. Retinas were washed again two times for 10 minutes with PBST and then once with PBS. Finally, retinas were placed on microscope slides photoreceptor side up, mounted with Vectashield mounting medium with 4',6diamidino-2-phenylindol (catalog number H-1200; Vector Laboratories, Burlingame, CA), and protected with coverslips.

Retinal whole mounts were imaged with a fluorescent microscope (Leica DMI6000B; Leica Microsystems Inc.) equipped with an automated stage. The microscope was set to scan the whole sample with a $10 \times$ objective and both visible (excitation $480 / 40 \mathrm{~nm}$ ) and far red (excitation 620/60 nm) filtered channels. Individual images were stitched together automatically with MetaMorph 7.8 software (Molecular Devices, Sunnyvale, CA) to form panorama retinal images.
The entire retinal area, $\mathrm{ONH}$ area, and damaged area were manually drawn with a MetaMorph software trace region tool. The damaged site was visually determined. Borders were drawn between locations showing normal cone outer segment morphology and density and locations where cone outer segments appeared shrunken with decreased density (Supplemental Fig. 1). The damaged area was determined for both S-opsin and M-opsin positive images by an observer blinded as to the experimental treatments. The percentage of damaged area compared with the whole retinal area $\mathrm{ONH}$ area subtracted) was used for statistical analyses.

Parallel High-Throughput Screening with the TANGO GPCR-Ome $\boldsymbol{\beta}$-Arrestin Recruitment Assay. Parallel TANGO high-throughput screening for agonist activity was performed as previously described (Kroeze et al., 2015). Human embryonic kidney 293-derived cells containing a stable tetracycline-controlled transcriptional activator (tTA)-dependent luciferase reporter and a $\beta$-arrestin 2-tobacco etch virus (TEV) fusion gene were cultured in Dulbecco's modified Eagle's medium with $10 \%$ fetal bovine serum, $2 \mu \mathrm{g} / \mathrm{ml}$ puromycin (Sigma-Aldrich), and $100 \mu \mathrm{g} / \mathrm{ml}$ hygromycin B (DiscoverX, Fremont, $\mathrm{CA}$ ) at $37^{\circ} \mathrm{C}$ in $5 \% \mathrm{CO}_{2}$ at $90 \%$ humidity. For transfection of 315 synthetic TANGO-ized GPCRs, cells were plated at $10^{6}$ cells per $150-\mathrm{mm}$ cell culture dish on day 1 . On day 2 , cells in each dish were transfected with a synthetic TANGO-ized GPCR (a total of 315 transfected cell lines) using a calcium phosphate method (Kroeze et al., 2015). On day 3, transfected cells were transferred at 20,000 cells/well in $40 \mu \mathrm{l}$ medium into poly(L-Lys) - coated, 384-well white clear-bottomed plates (Greiner Bio-One, Kremsmünster, Austria) in quadruplicate. Control cells transfected with DRD2 were seeded as 16 replicates in columns 1 and 2 . On day 4, four single drug stimulation solutions (of either MTP, TAM, DOX, or BRM) and six combined drug stimulation solutions (of either 1:1 MTP + DOX, 1:1 MTP + TAM, 1:1 MTP + BRM, 1:1 DOX + BRM, 1:1 TAM + BRM, 1:1: $1 \mathrm{MTP}+\mathrm{DOX}+\mathrm{BRM}$, and 1:1:1 MTP + TAM + BRM) were prepared in filter-sterilized assay buffer and $20 \mu \mathrm{l}$ were added to each well to achieve a final concentration of $10 \mu \mathrm{M}$. For the control containing 16 replicates in column $1,100 \mathrm{nM}$ quinpirole drug stimulation solution was added, whereas no ligand was added in column 2 . On day 5 , drugcontaining medium was removed from the wells and $20 \mu \mathrm{l}$ Bright-Glo solution (Promega, Madison, WI) diluted 20-fold with assay buffer was added to each well. After incubation for 20 minutes at room temperature, luminescence was measured with Trilux (Trilux, Arnsberg, Germany). GraphPad Prism software (GraphPad Inc., La Jolla, CA) was used for data analyses.

RNA-Seq Transcriptome Analyses of the Eye and Retina. RNA-seq transcriptome analyses of the human retina were performed as previously described (Mustafi et al., 2013b). Mouse retina RNA was isolated with TRIzol (Invitrogen, Carlsbad, CA) and libraries were generated from $100 \mathrm{ng}$ total RNA with a TruSeq Stranded mRNA Library Prep Kit (Illumina, San Diego, CA). Base paired-end sequence reads (125) were obtained on the HiSEq 2500 platform (Illumina). Raw reads were initially analyzed with Illumina software and mapped to the GRCm38.p3/Ensembl v78 assembly with the TopHat2 annotation (version 2.1.0). Mono- and combined drug-treated $A b c a 4^{-1-} R d h 8^{-/-}$ or BALB/c mice were light exposed for 24 hours, as described previously (Chen et al., 2016). Doses used for monotherapy were $10 \mathrm{mg} / \mathrm{kg}$ b.wt. for MTP, $2.5 \mathrm{mg} / \mathrm{kg}$ b.wt. for TAM, $10 \mathrm{mg} / \mathrm{kg}$ b.wt. for DOX, and $1 \mathrm{mg} / \mathrm{kg}$ b.wt. for BRM. Doses used for combined treatment with MTP + TAM + BRM were set to $0.1 \mathrm{mg} / \mathrm{kg}$ b.wt. for BRM, $1 \mathrm{mg} / \mathrm{kg}$ b.wt. for MTP, and $0.05 \mathrm{mg} / \mathrm{kg}$ b.wt. for TAM. Doses used for combined treatment with MTP + DOX + BRM were set to $1 \mathrm{mg} / \mathrm{kg}$ b.wt. for MTP, $1 \mathrm{mg} / \mathrm{kg}$ b.wt. for DOX, and $0.1 \mathrm{mg} / \mathrm{kg}$ b.wt. for BRM. Transcripts expressing $\geq 1.0$ fragments per kilobase of transcript per million mapped reads in all samples were further evaluated, and effective counts from the eXpress output of filtered fragments per kilobase of transcript per million mapped reads were normalized and statistically evaluated (Chen et al., 2016).

Real-Time PCR of GPCRs That Are Targets for Combinatorial Treatment. Human retina cDNA was purchased from Clontech 
(catalog no. 637216; Mountain View, CA). This cDNA was derived from normal retinas pooled from 99 male and female Caucasians aged $50-80$ years who had died suddenly. The cDNA was diluted 1:10 with water. The PCR premixture was prepared with PowerUp SYBR Green Master Mix (Applied Biosystems), human retina cDNA, and water and then aliquoted into 15 tubes. Primers were added to each tube, resulting in a $0.3 \mu \mathrm{M}$ final concentration. Detailed primer sequences are listed in Supplemental Table 1. After mixing and brief centrifugation, triplicate aliquots of $12 \mu \mathrm{l}$ from each sample were transferred into three adjacent wells of a 96 -well plate. PCR conditions were set as follows: heat at $95^{\circ} \mathrm{C}$ for 3 minutes; run 45 cycles at $95^{\circ} \mathrm{C}$ for 15 seconds and at $60^{\circ} \mathrm{C}$ for 1 minute. The melting curve was tested by heating at $95^{\circ} \mathrm{C}$ for 15 seconds, at $55^{\circ} \mathrm{C}$ for 15 seconds, and slowly annealing up to $95^{\circ} \mathrm{C}$ during a 20 -minute period. The PCR machine used for this experiment was a Mastercycler RealPlex2 (Eppendorf AG, Hamburg, Germany).

Measurements of Combination Drug Therapy Effects on ERG Function in Wild-Type Mice. Before drug administration and ERG measurements, 4-week-old C57BL/6J mice were placed in a dark room for at least 12 hours. Treatments consisted of MTP, DOX, and BRM; MTP, TAM, and BRM; or DMSO alone. All treatment procedures were performed in a dark room. Drugs or DMSO were administered by intraperitoneal injection ( $50 \mu \mathrm{l}$ ) either 45 minutes or 3 hours before ERG measurements. Five mice were used for each group.

Drug combinations and doses were as follows: 1) $1 \mathrm{mg} / \mathrm{kg}$ b.wt. MTP, $1 \mathrm{mg} / \mathrm{kg}$ b.wt. DOX, and $0.1 \mathrm{mg} / \mathrm{kg}$ b.wt. BRM, all in DMSO; and 2) $1 \mathrm{mg} / \mathrm{kg}$ b.wt. MTP, $0.05 \mathrm{mg} / \mathrm{kg}$ b.wt. TAM, and $0.1 \mathrm{mg} / \mathrm{kg}$ b.wt. BRM; all in DMSO. The control consisted of DMSO alone.

Scotopic and photopic ERG measurements were obtained as previously described (Chen et al., 2013). Briefly, dark-adapted mice were anesthetized with $20 \mathrm{mg} / \mathrm{ml}$ ketamine and $1.75 \mathrm{mg} / \mathrm{ml}$ xylazine in PBS at a dose of $0.1-0.13 \mathrm{ml} / 25 \mathrm{~g}$ b.wt. After anesthesia, pupils were dilated with $1 \%$ tropicamide. Contact lens electrodes were placed on the eyes and a reference electrode and ground electrode were positioned between two ears and on the tail. Scotopic and photopic ERGs were recorded from both eyes of each mouse with a UTAS E-3000 universal testing and ERG system (LKC Technologies Inc., Gaithersburg, MD) either 45 minutes or 3 hours after treatment with a drug combination or DMSO. ERG data represent the means \pm S.D. of both a-wave and b-wave amplitudes derived from measurements of 10 eyes per group, either 45 minutes or 3 hours after drug treatment.

Pharmacokinetics: Routes of Drug Administration. Lower doses of drugs were administered in this study than previously described (Chen et al., 2016), requiring the use of detection methods that offered higher sensitivity. However, this gain in sensitivity came with other complications such as the requirement for high quality standards modified with stable isotopes and regular tests of drug stability. Biologic samples underwent no purification procedures other than organic phase extraction to maintain their near native levels of drugs. In designing the tuning method, the same tune parameters were employed for MTP, TAM, and DOX. A special file was created for BRM by its manual injection and optimizing for the main ion $(\mathrm{m} / z$ 656.70). The tune method used for MTP, TAM, and DOX was produced in a similar manner. It was unnecessary to create separate tune files for these drugs. This approach provided considerable optimization, especially for samples involving multiple drug treatments. Thus, more than one drug could be quantified in the same run.

Intraperitoneal Injections. MTP, TAM, DOX, and BRM were dissolved to their specified concentrations in DMSO. Manually restrained mice were injected with $50 \mu \mathrm{l}$ solubilized drugs while the head and body were tipped downward to move the viscera away from the ventral abdominal surface. Injections were directed away from the midline into the lower right abdominal quadrant to avoid injuring the urinary bladder and cecum.

Gastric Gavage. Drugs administered by gastric gavage (catalog number 18060-20m 20-gauge needle, 1.25-mm optical density barrel tip $\times 30 \mathrm{~mm}$; Fine Science, Foster City, CA) were dissolved in PBS. The solution volume administered was $50 \mu \mathrm{l}$ for all treatments.
Perfusion Procedures. Mice were anesthetized in a carbon dioxide chamber before the abdominal and chest cavities were opened. The heart was exposed before a small cut was made on the left ventricle using scissors. A perfusion needle was inserted through the ventricle into the root of the aorta and was then clamped with a hemostat. After cutting the right atria to release blood from the vena cava, perfusion started at a flow rate of $18 \mathrm{ml} / \mathrm{min}$ for 2 minutes with a $37^{\circ} \mathrm{C}$ saline solution. Each perfusion was performed with a pressure infuser apparatus (Infusurge, 4010; Ethox, Buffalo, NY) connected to a catheter set with a regulating clamp $(2 \mathrm{C} 5417 \mathrm{~s}, 70$ inches; Baxter, Deerfield, IL) and a perfusion needle (catalog number 18060-20, 20 -gauge, 1.25 -mm optical density barrel tip $\times 30 \mathrm{~mm}$; Fine Science). The perfusion time was 2 minutes. Perfusion completion was checked by visual inspection of the brain and tongue, which were devoid of blood.

Quantification of MTP, TAM, DOX, and BRM Levels in Serum and Eyes of Mice after Treatments. Blood was collected from treated mice and allowed to clot at $22^{\circ} \mathrm{C}$ for 20 minutes. Clotted blood samples were centrifuged for 10 minutes at $4000 \mathrm{~g}$ in a temperature-controlled benchtop centrifuge (Eppendorf AG). Serum $(150 \mu \mathrm{l})$ was carefully removed to avoid disturbing loose clots. Samples were stored at $-80^{\circ} \mathrm{C}$ until further processing. For analyses, each solution was supplemented with $10 \mu \mathrm{l} \mathrm{NH} \mathrm{N}_{4} \mathrm{OH}$ and incubated at $22^{\circ} \mathrm{C}$ for 10 minutes. Samples were centrifuged at 50,000g for 1 hour at $4^{\circ} \mathrm{C}$ in an Optima MAX benchtop ultracentrifuge (Beckman-Coulter, Indianapolis, IN). Supernatants were removed and placed in disposable 13- $\times 100-\mathrm{mm}$ culture tubes made of borosilicate glass (Fisher Scientific). Four microliters of the appropriate internal standard $(100 \mu \mathrm{g} / \mathrm{l})$ was added to each sample (see Supplemental Table 2).

Samples from combination drug treatments were processed as just described and specific dilutions of the final sample solution were used, as presented in Supplemental Table 2.

Organic Solvent Extraction of Drugs. Samples were next extracted with $3 \mathrm{ml}$ methyl tert-butyl ether (Sigma-Aldrich). Mixing was achieved by withdrawing $1 \mathrm{ml}$ of sample and pipetting it back into the remaining volume, a procedure repeated 15 times. Samples then were centrifuged for 15 minutes at $100 \mathrm{~g}$ to separate the organic and aqueous layers. Next, the organic layer was removed and dried under a gentle stream of argon gas. After the organic solvent was evaporated, the bottom of the glass tube was washed with $150 \mu \mathrm{l}$ methanol/water $[50: 50(\mathrm{v} / \mathrm{v})]$, making sure the walls were washed from the bottom of the tube up to $2 \mathrm{~cm}$ in height. Next, this solution, denoted as the "final sample solution" in Supplemental Table 2, was removed, placed in a microfuge polypropylene tube, and centrifuged at 50,000 $\mathrm{g}$ for 1 hour at $4^{\circ} \mathrm{C}$ to remove insoluble material. The final amount of each sample to be injected was initially evaluated by a trial injection to ensure that the response would be in the linear range of the concentration as a function of the ion intensity established for each tested drug. Optimum injection volumes were achieved through dilution. These values are detailed for each drug/drug combination dose in Supplemental Table 2.

Liquid Chromatography/Mass Spectrometry Analyses. A specific volume of each sample (see Supplemental Table 2) was injected onto a Luna 5- $\mu \mathrm{m}$ C8 100-Å column (Phenomenex, Torrance, CA) previously equilibrated with water containing $0.1 \%$ aqueous formic acid. The injection was performed with a temperaturecontrolled autosampler at $4^{\circ} \mathrm{C}$ coupled to a high-performance liquid chromatography (LC) unit at a flow rate of $0.1 \mathrm{ml} / \mathrm{min}$. Column outflow was introduced into an LTQ mass spectrometer (ThermoFisher Scientific, Waltham, MA) equipped with an electrospray ionization unit operating at $300 \mathrm{~K}$. Chromatographic conditions were as follows: 0-5 minutes, $98 \% \mathrm{~A}$ (water in $0.1 \%$ aqueous formic acid) and $2 \% \mathrm{~B}$ (acetonitrile in $0.1 \%$ formic acid); $5-18$ minutes, a linear gradient to $2 \% \mathrm{~A}$ and $98 \% \mathrm{~B}$; and $18-20$ minutes, $98 \% \mathrm{~A}$. Only the eluent between 10 and 14 minutes was diverted into the mass spectrometer. The liquid LC-mass spectrometry (MS) method was established as a selected reaction monitoring system for two ions: the drug and its designated internal standard (see Supplemental Fig. 4 for details). 
Quantification was done automatically by evaluating the area under the curve for the peak that eluted at a specific time and region (see details in Supplemental Fig. 4, D, H, L, and P).

Preparation of Final Sample Solutions from Eyes. After mice were euthanized, eyes were removed and kept frozen on dry ice until they were transferred to a $-80^{\circ} \mathrm{C}$ environment prior to processing. Two frozen eyes were used per sample. Samples were then sonicated (QSonica, Newton, CT) in $200 \mu \mathrm{l}$ methanol/water [50:50 (v/v)] by four repeats of 10-second pulses per repeat with an amplitude set to $75 \%$. Solutions were supplemented with $10 \mu \mathrm{l} \mathrm{NH} \mathrm{NH}_{4} \mathrm{OH}$ and incubated at $22^{\circ} \mathrm{C}$ for 10 minutes. Samples were centrifuged at $50,000 \mathrm{~g}$ for 1 hour at $4^{\circ} \mathrm{C}$ in an Optima MAX benchtop ultracentrifuge. Supernatants were placed in 13- $\times 100$-mm disposable culture tubes made of borosilicate glass (Fisher Scientific).

Statistical Analyses. Two-way analysis of variance was applied to the analysis of ONL thickness and ERG measurements. Analyses of variance were followed by Tukey's multiple comparison tests. The analysis of retinal damage area was accomplished with a nonparametric Kruskal-Wallis test, followed by Dunn's multiple comparison tests. The data are presented as means \pm S.E.M. The level of statistical significance was set at $P<0.05$.

\section{Results}

Cone Photoreceptors Are Protected by a Combined Therapy with MTP, TAM, and BRM. We investigated the efficacy of MTP + TAM + BRM (10, 0.5, and $1 \mathrm{mg} / \mathrm{kg}$, respectively) combined treatment in mitigating the effects of acute, bright light-induced retinal degeneration in an $A b c a 4^{-1-} R d h 8^{-l-}$ mouse model of Stargardt disease. Since we previously demonstrated a positive effect of this combination therapy on rod photoreceptors (Chen et al., 2016), this investigation focused on cone cell survival, especially since cone cells are critical for the normal daily activities of humans. To assess the pharmacokinetics of these drugs, different durations were examined between drug injection and the initiation of BLE. Thus, drugs were injected either $0.5,2$, or 4 hours prior to BLE. The effects of drug treatment were evaluated by OCT to determine the structural integrity of photoreceptors as assessed by the thickness of the retinal ONL and ERG to assess cone photoreceptor function. The area of retinal damage following BLE was measured by analyzing

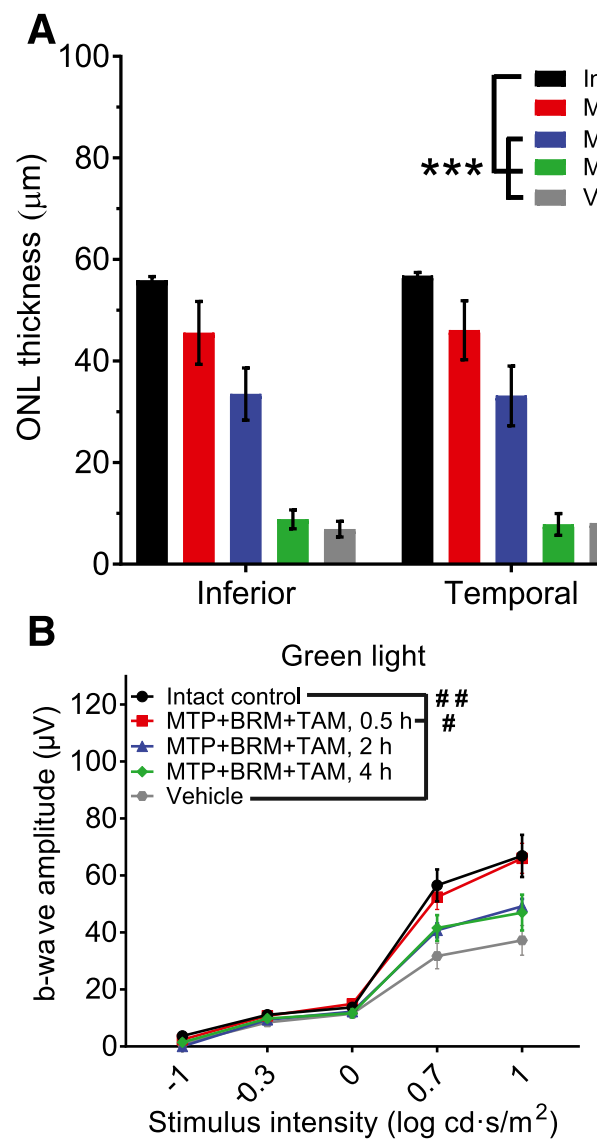

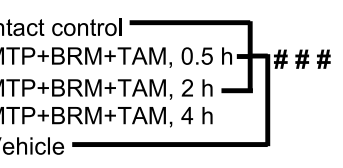
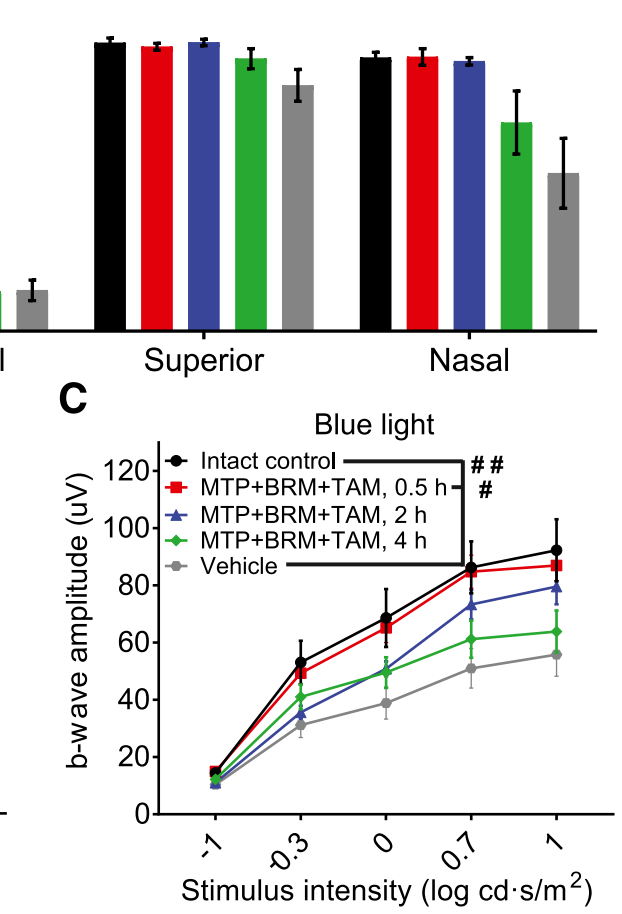

D

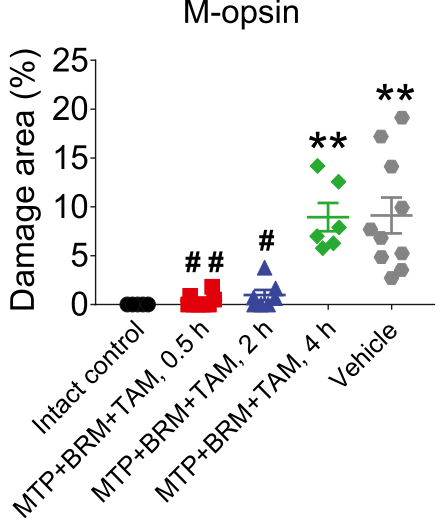

E

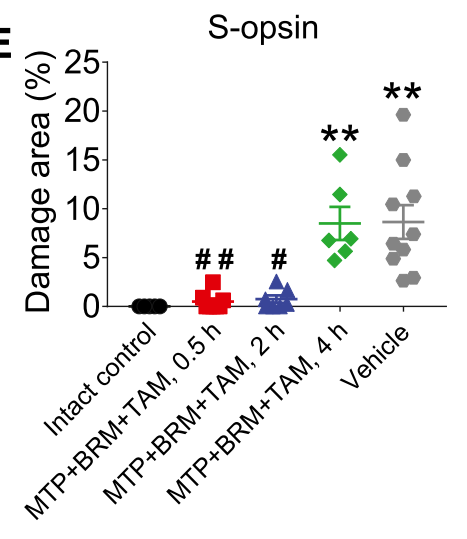

Fig. 1. Protection of retinal photoreceptor cells with MTP + TAM + BRM pretreatment. A single intraperitoneal injection of MTP + TAM + BRM protects retinal photoreceptor cells when given 0.5-2 hours prior to BLE (0.5 hours at 25 klux, $6500 \mathrm{~K}$ light). (A) Thickness of the ONL. ONL thickness was measured from in vivo OCT images (see Supplemental Fig. 1 for illustration) $500 \mu \mathrm{m}$ from the ONH. Treatment with MTP + TAM + BRM prevented ONL thinning completely when given 0.5 hour prior to BLE (control versus MTP + TAM + BRM, 0.5 hour; $P=0.18$ ) and partially when injected 2 hours prior to BLE, but not when these drugs were given 4 hours prior to BLE. (B and C) Treatment with MTP + TAM + BRM prevents retinal dysfunction as assessed by measuring green light (B) and blue light (C) photopic ERG responses. (D and E) Retinal damage area analysis. The damaged area was measured from M-opsin and S-opsin stained retinal whole-mount images (see Supplemental Fig. 2 for examples from each group). Statistical analyses were performed with two-way ANOVA (OCT), with repeated-measures two-way ANOVA (ERGs), or with the Kruskal-Wallis test (damaged area analyses). ANOVAs were followed with Tukey's post hoc test, and the Kruskal-Wallis test was followed with Dunn's post hoc test. Asterisks indicate statistically significant differences compared with control mice $\left({ }^{* *} P<0.01 ;{ }^{* * *} P<0.001\right)$ and pound signs compared with vehicle-treated mice $\left({ }^{\#} P<0.05\right.$; ${ }^{\# \#} P<0.01 ;{ }^{\# \# \#} P<$ 0.001). Group sizes in all analyses were as follows: intact control, $n=9$; MTP + TAM + BRM 0.5 hour, $n=8$; MTP + TAM + BRM 2 hours, $n=7$; MTP + TAM + BRM 4 hours, $n=6$; and vehicle, $n=10$. Data are presented as means \pm S.E.M. ANOVA, analysis of variance. 
retinal flat mounts labeled with S-opsin and M-opsin antibodies. Exposure to bright light induced a marked reduction in ONL thickness in vehicle-treated mice, especially in the infratemporal retina (Fig. 1A; Supplemental Fig. 1), thus demonstrating the loss of unprotected photoreceptor cells. Also observed was a moderate decline of photopic ERG amplitudes (Fig. 1, B and C) and a drastic loss and/or disorganization of S-cones and M-cones in the central but not the peripheral retina (Supplemental Fig. 1).

MTP + TAM + BRM fully protected the retinas in most mice when this combination was given IP 0.5 hour prior to BLE (Fig. 1; Supplemental Table 3); there were no statistically significant differences between drug-treated and control mice as measured by ONL thickness, photopic ERG amplitudes or degree of cone cell damage. When drugs were given 2 hours prior to BLE, fewer mice were fully protected as indicated by their ONL thickness (Supplemental Table 3). Instead, most mice showed intermediate ONL protection, and the photopic ERG response amplitudes appeared slightly reduced compared with controls (Fig. 1, B and C). Interestingly, both S-cone and M-cone photoreceptors were well preserved in most mice that were drug-injected 2 hours prior to BLE (Fig. 1, $\mathrm{D}$ and E; Supplemental Table 3), indicating that BLE induces less damage to cones than rods in $A b c a 4^{-/-} R d h 8^{-1-}$ mice.
A

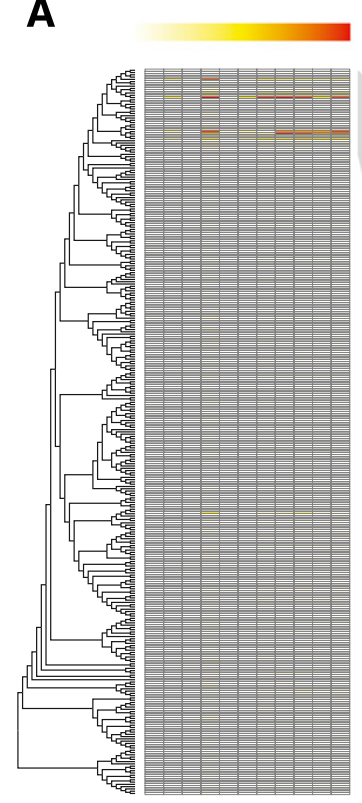

B
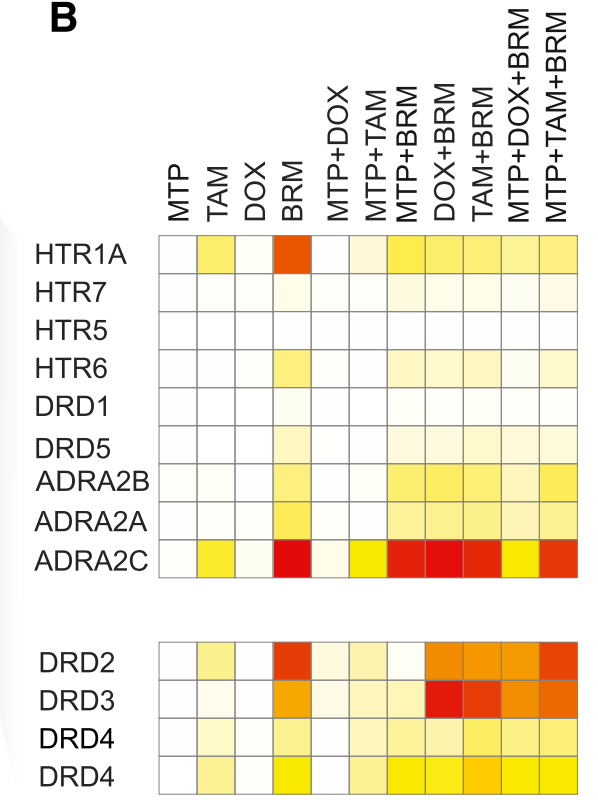

C
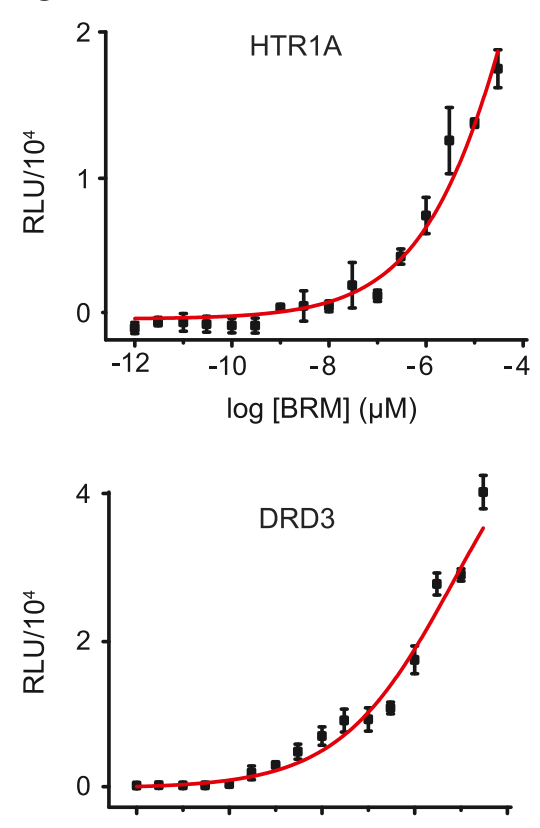

$\log [\mathrm{BRM}](\mu \mathrm{M})$
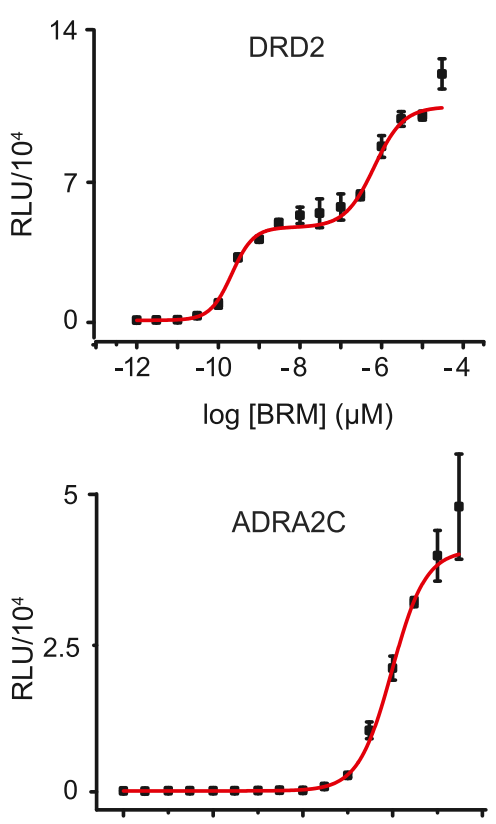

$\log [\mathrm{BRM}](\mu \mathrm{M})$
Fig. 2. Agonistic activity of MTP, TAM, DOX, and BRM toward a GPCR panel. (A) PRESTO-TANGO (Parallel Receptor-ome Expression and Screening via Transcriptional Output-TANGO) screen of four known GPCRtargeted drugs (MTP, TAM, DOX, and BRM). These drugs were screened in 11 different combinations for their constitutive agonistic activity against 315 orphan and poorly annotated GPCRs (University of North Carolina collection). (B) Screening of multiple targets simultaneously in a parallel fashion. (C) Dose-dependent trials. These trials used the same system and 16 drug concentrations ranging from $0.001 \mathrm{nM}$ to $100 \mu \mathrm{M}$. BRM dose-dependent values for HTR1A, DRD2, DRD3, and ADRA2C receptors were determined. RLU, relative luminescence unit. 
Finally, when drugs were injected 4 hours prior to BLE, no retinal protection was observed in any of the treated mice (Supplemental Table 3).

Specificity of GPCR Modulators toward Receptor Activation. An unbiased cellular assay was next used to investigate the agonistic-like activity of MTP, TAM, DOX, and BRM toward a comprehensive group of GPCRs. The TANGO assay monitors the activation of GPCR-generated fusion proteins with a cleavable membrane localization signal, a cell surface expressed FLAG tag, a TEV cleavage site, and tTA protein (Barnea et al., 2008). Ligand binding to a targeted GPCR receptor stimulates recruitment of a $\beta$-arrestin 2 -TEV protease fusion, triggering the release of the tethered transcription factor tTA that then stimulates reporter gene activity. PRESTO-TANGO (Parallel Receptor-ome Expression and Screening via Transcriptional Output) was used to screen the four known GPCR-targeted drugs under investigation here (MTP, TAM, DOX, and BRM) in 11 different combinations for their agonist activity toward 315 orphan and poorly annotated GPCRs (Fig. 2A). Screening multiple targets simultaneously with mono- and combined drug treatments revealed BRM-dependent agonistic activation of the GPCRs serotonin receptor 1A (HTR1A), DRD2, DRD3, and adrenergic receptor subtype $\mathrm{A} 2 \mathrm{C}$ (ADRA2C) (Fig. 2B). In contrast, the analysis of MTP (an antagonist of $\mathrm{G}_{\mathrm{s}}$-coupled $\beta_{1}$-ARs), TAM (an antagonist of $\mathrm{G}_{\mathrm{q}}$-coupled $\alpha_{1}$-Ars), and DOX (an antagonist of $\mathrm{G}_{\mathrm{q}}$-coupled $\alpha_{1}$-ARs) showed no significant effect on GPCR activation, as expected, confirming that these compounds act solely as GPCR antagonists (Chen et al., 2016). Dosedependence trials using the same system with 16 different concentrations of BRM ranging from $0.001 \mathrm{nM}$ to $100 \mu \mathrm{M}$ were then tested. HTR1A, DRD2, DRD3, and ADRA2C demonstrated $\mathrm{EC}_{50}$ values of $330 \mu \mathrm{M}, 31$ and $670 \mathrm{nM}$ (accounting for the dual-binding properties of DRD2), and 0.7 and $1 \mu \mathrm{M}$, respectively (Fig. 2C).

Expression of GPCRs in Human Retina. We measured the expression of different GPCRs in the human retina, and we also determined whether these expression levels were altered by the four known GPCR-targeted drugs under investigation in this study. Both RNA-seq and quantitative polymerase chain reaction (qPCR) analyses were carried out to achieve this objective. Initially, RNA-seq and qPCR were performed using a single human donor retina, and then qPCR also was carried out with a retinal library generated from 99 human patients. Primer sequences are shown in Supplemental Table 1. Expression levels of 13 GPCRs demonstrated in a previous study as potential pharmacological targets of the four drugs under investigation here (Chen et al., 2016) were detected in the human retina by qPCR in both experiments (Supplemental Table 4). To compare the qPCR data with human RNA-seq data (Mustafi et al., 2016), all GPCR expression levels were arbitrarily normalized to that of tachykinin receptor 1 (Tacr1) (assigned the value of 1). When qPCR results were compared between the single donor and pooled donors (Supplemental Table 4), most GPCR expression levels were comparable, except for glutamate metabotropic receptor 4 and prostaglandin $\mathrm{E}$ receptor 1 . Together, the comparison of results from RNA-seq and qPCR studies revealed that the measured GPCR levels are highly conserved.

Next, because drugs taken orally have the potential to affect the expression levels of GPCRs in tissues other than the retina, we also measured GPCR expression in the whole eye compared with that of the retina. Furthermore, since different animal models are used to evaluate drug therapies for the treatment of retinal degenerative diseases, a comprehensive comparison of GPCR expression in the eye and retina of different species was conducted. RNA-seq analyses were conducted to compare retinas and whole eyes from various species including the mouse (a rod-dominant species) (Jeon et al., 1998), Nile rat (similar to the human retina in its $\mathrm{rod} /$ cone ratio) (Mustafi et al., 2016), ground squirrel (a conedominant retina) (Anderson and Jacobs, 1972; Szél et al., 1993), and monkey and human retina (Mustafi et al., 2016) (see Table 1). The mouse study employed C57BL/6 wild-type mice, 3-month-old rhodopsin knockout C57BL/6 mice that lack both rods and cones (Humphries et al., 1997; Lem et al., 1999), and 4-week-old neural retina-specific leucine zipper protein (Nrl) knockout mice with a C57BL/6 background that develop uniform cone-like photoreceptor cells (Bessant et al., 1999; Mears et al., 2001; Mustafi et al., 2011).

Comparisons showed that expression levels of most GPCRs were consistent among species, whereas some GPCRs (e.g., prostaglandin E receptor 1) appeared to be highly expressed in rodents but less in monkey and human retinas. Drd4 was the most abundant GPCR expressed in all species, except the Nile rat (Table 1). But Drd4 expression levels dropped significantly in Rho and $\mathrm{Nrl}$ knockout mice compared with wild-type mice indicating its expression in rod cells. The expression of the adenosine $\mathrm{A} 2 \mathrm{~b}$ receptor increased in rod-deficient mice, and its increase in cone-dominant retinas suggested at least partial expression in cone cells. $\beta_{1}$-ARs increased significantly in $\mathrm{Nrl}$ knockout mouse retinas, indicating their expression in cone photoreceptors.

To examine the impact of MTP, TAM, DOX, and BRM monoor combined drug treatments on the expression of all GPCRs in the retina, a global transcriptome analysis by RNA-seq was performed. Retinas were collected from mice that had not been exposed to bright light and from pretreated mice (either with DMSO or individual or combined drugs) 1 day after exposure to light. First, the reduction of opsin expression in control mice exposed to bright light was mitigated by single drug treatment with either MTP, TAM, or DOX and BRM (Fig. 3), further supporting that these GPCR modulators help preserve photoreceptor cells. Next, the bright light-induced expression of Endothelin receptor type B, neuropeptide Y receptor Y4, GPCR126, and GPR146 was attenuated by the same treatments (Fig. 3). Combination treatments with MTP + TAM + $\mathrm{BRM}$ or MTP + DOX + BRM resulted in sequence expression profiles similar to drugs used individually although drug levels used in the combination studies were much lower than those used in the monotherapy studies (Fig. 3). Cellular localization of these receptors was not determined.

Collectively, these data demonstrate that 13 GPCRs demonstrated in a previous study as potential pharmacological targets of the four drugs investigated in this study (Supplemental Table 4) and in a prior study (Chen et al., 2016) are expressed in the human retina. Furthermore, the expression of these receptors is conserved across species. However, there are differences in the expression levels of some GPCRs. Moreover, MTP, TAM, DOX, and BRM did not significantly affect the expression of GPCRs targeted by the four drugs under investigation in this study, nor did they have demonstrable effects on the expression of other receptors present in the eye. Therefore, these data indicate that the combination 
TABLE 1

Comparison of retina GPCR gene expression levels in different species by RNA-seq

\begin{tabular}{|c|c|c|c|c|c|c|c|c|}
\hline \multirow{2}{*}{ Gene } & \multicolumn{3}{|c|}{ C57BL/6 Mouse } & \multirow{2}{*}{ Rat } & \multirow{2}{*}{ Nile $\operatorname{Rat}^{a}$} & \multirow{2}{*}{ Ground squirrel } & \multirow{2}{*}{ Monkey } & \multirow{2}{*}{ Human } \\
\hline & WT & Rho KO & $\mathrm{Nrl} \mathrm{KO}$ & & & & & \\
\hline Drd1a & 6.1 & 5.89 & 15.60 & 18.7 & 13.4 & 15.8 & 12 & 7.9 \\
\hline Drd4 & 229.5 & 39.6 & 23 & 110.8 & 8.2 & 21.1 & 165 & 126 \\
\hline Drd2 & 20.9 & 17 & 14.3 & 30.9 & 4.2 & 7.2 & 22.4 & 24.8 \\
\hline Crhr1 & 6 & 5.3 & 2.8 & 7.9 & 1.1 & 0.9 & 2 & 5.2 \\
\hline Grm1 & 2.7 & 1.92 & 3 & 3 & 1.2 & 2.3 & 3.2 & 4.7 \\
\hline Adrb1 & 19.9 & 12.3 & 94.2 & 26.8 & 7.8 & 1.4 & No homolog & 3.4 \\
\hline Adora $2 \mathrm{~b}$ & 2.5 & 9 & 1.5 & 1.1 & 7.9 & 6.8 & 16.2 & 3.4 \\
\hline Grm4 & 4.7 & 6.3 & 1.7 & 5.6 & 1.8 & 5.4 & No homolog & 1.9 \\
\hline Adra1a & 0.3 & 0.6 & 0.1 & 0.8 & ND & ND & 0.9 & 0.2 \\
\hline Adra1b & 1.6 & 2.3 & 0.6 & 4.2 & 1 & 0.4 & 2.8 & 1 \\
\hline Adra1d & 3.8 & 4.3 & 2.1 & 1 & ND & 2.5 & 2.3 & 0.1 \\
\hline Tacr1 & 2 & 5.1 & 3 & 4.9 & 1.8 & 0.8 & 1 & 0.9 \\
\hline Ptger1 & 14.1 & 17 & 3.9 & 9.6 & 4.9 & 4.8 & 0.5 & 0.8 \\
\hline
\end{tabular}

Adora2b, adenosine A2b receptor; Adrb, beta-1 adrenergic receptor; Adra1a, alpha-1a adrenergic receptor; Adra1b, alpha-1b adrenergic receptor; Adra1d, alpha-1d adrenergic receptor; Crhr1, corticotropin releasing hormone receptor 1; Drd1, dopamine receptor D1; Drd2, dopamine receptor D2; Drd4, dopamine receptor D4; Grm1, glutamate metobatrophic receptor 1; Grm4, glutamate metobatrophic receptor 4; Ptger1, prostaglandin E receptor 1; Tacr1, tachykinin receptor 1.

${ }^{a}$ RNA isolated from the whole eye.

therapy that protects photoreceptors in a mouse model of retinal degeneration could be relevant in treating human conditions, since the targeted receptors are constituents of the mammalian eye/retina but their levels of expression appear unaltered by drug treatment, lessening the potential for undesired effects in other cell types.

Effects of Drugs on ERG Responses. To ensure that drug treatments do not interfere with the normal processing of light signals in the retina, the impact of MTP $(1 \mathrm{mg} / \mathrm{kg})$, TAM $(0.05 \mathrm{mg} / \mathrm{kg})$, DOX (1 mg/kg), and BRM $(0.1 \mathrm{mg} / \mathrm{kg})$ and their combinations was also investigated on retinal function in wildtype mice unexposed to damaging light. ERG responses were recorded in 4-week-old wild-type C57BL/6 mice treated with drug combinations or DMSO, and the results of two combinations are shown (MTP, TAM, and MTP or MTP, DOX, and $\mathrm{BRM})$. Treatments were administrated to mice under dim red light by intraperitoneal injection either 45 minutes or 3 hours prior to ERG recordings. Both scotopic and photopic b-wave ERG data are summarized in Fig. 4 and a-wave ERG data are summarized in Supplemental Fig. 3. ERG recordings revealed that both a-wave and b-wave amplitudes were of similar magnitudes in mice treated with DMSO or drug combinations that target multiple GPCRs whether taken 45 minutes or 3 hours after drug administration. There were no significant differences in ERG amplitudes among the three groups (two combination therapy groups and one vehicle control group), indicating that the combination pretreatments did not adversely affect visual function.

Pharmacokinetics of GPCR Modulators. To learn about their pharmacokinetic profiles in the context of retinal protection, MTP, TAM, DOX and BRM levels were measured in mouse sera and eyes after single dose administration of these drugs.
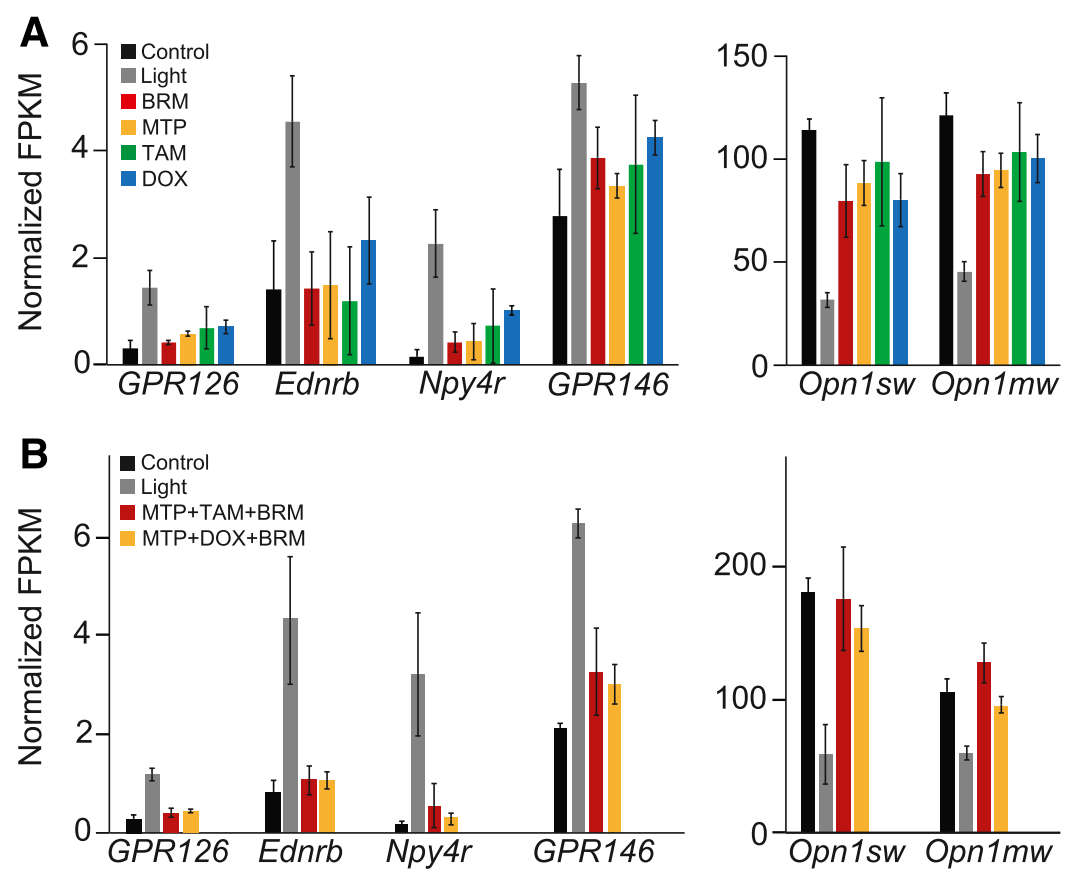

Fig. 3. RNA-seq analysis of retinal gene expression. RNAseq experiments were performed on retinal samples collected from $A b c a 4^{-1-} R d h 8^{-1-}$ mice. Two groups were compared: one unexposed to bright light (control) and lightexposed mice pretreated with DMSO (Light) or with either MTP, TAM, and BRM or MTP, DOX, and BRM $(n=$ 3/group). (A) Normalized fragments per kilobase of genes after single drug treatments. Total RNA was subjected to RNA-seq analysis, and FPKM, normalized FPKM, were calculated. (B) Normalized fragments per kilobase of genes after combined drug treatments. Combined drug treatments consisted of either MTP + TAM + BRM or MTP + DOX + BRM. Drug doses for monotherapy were as follows: $10 \mathrm{mg} / \mathrm{kg}$ MTP, $2.5 \mathrm{mg} / \mathrm{kg}$ TAM, $10 \mathrm{mg} / \mathrm{kg}$ DOX, and $1 \mathrm{mg} / \mathrm{kg}$ BRM. Doses for combination therapy were as follows: $1 \mathrm{mg} / \mathrm{kg}$ MTP, $0.05 \mathrm{mg} / \mathrm{kg}$ TAM, $1 \mathrm{mg} / \mathrm{kg}$ DOX, and $0.1 \mathrm{mg} / \mathrm{kg}$ BRM. FPKM, fragments per kilobase of transcript per million mapped reads. 

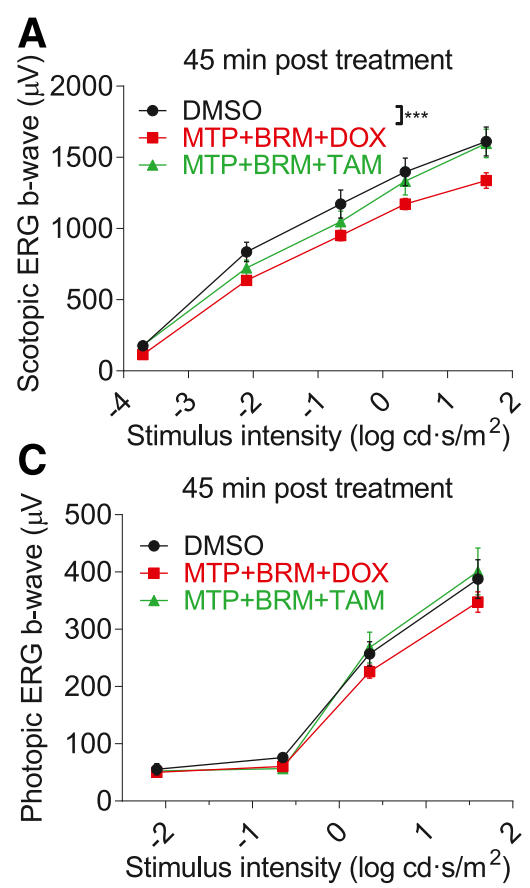
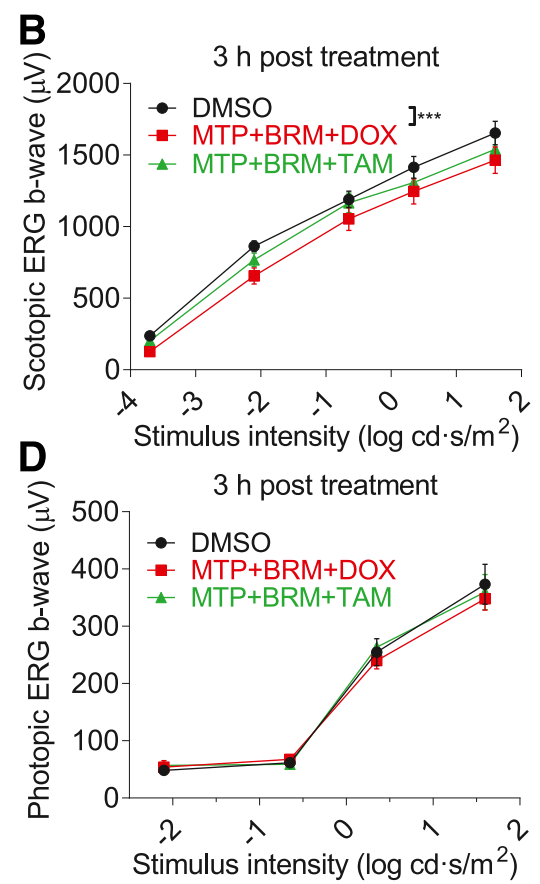

Fig. 4. Physiologic testing of the effects of GPCR drugs on retinal function. Two drug combinations $[1 \mathrm{mg} / \mathrm{kg}$ MTP, $1 \mathrm{mg} / \mathrm{kg}$ DOX, and $0.1 \mathrm{mg} / \mathrm{kg}$ BRM $(\mathrm{MTP}+\mathrm{DOX}+\mathrm{BRM})$ or $1 \mathrm{mg} / \mathrm{kg}$ MTP, $0.05 \mathrm{mg} / \mathrm{kg}$ TAM, and $0.1 \mathrm{mg} / \mathrm{kg}$ BRM (MTP + $\mathrm{TAM}+\mathrm{BRM}$ )] were administrated to 4 -week-old C57BL/6J mice. ERG recordings (b-waves) were compared between groups treated with either drug combinations or DMSO. (A) ERG responses were recorded to evaluate the impact of these drug combinations on retinal function in wild-type mice at 45 minutes post-treatment under scotopic conditions. (B) ERG responses recorded 3 hours after drug administration under scotopic conditions. (C) ERG responses also were recorded to evaluate the impact of drug combinations on retinal function in wild-type mice at 45 minutes after drug administration under photopic conditions. (D) ERG responses recorded 3 hours after drug administration under photopic conditions. ERG recordings showed that there were no significant differences in b-wave amplitudes among the three groups (two groups treated with drug combinations and one group treated with DMSO). Data are shown as means \pm S.E.M. $(n=10$ eyes $)$. $* * * P<$ 0.001 , analysis of variance.
MTP fragmentation produced two main ions: $\mathrm{m} / z 116.08$ and $m / z 191.08$ (Supplemental Fig. 4A). The internal standard chosen for MTP was labeled with seven deuterium atoms, MTP-d7 (Supplemental Fig. 4B). For both MTP and MTP-d7, the ion with $\mathrm{m} / z 191$ corresponds to fragmentation of the N-C imine bond (Supplemental Fig. 4, A and B) followed by loss of water $(-18)$ and $\mathrm{NH}_{3}(-17)$. Although $\mathrm{m} / z 191$ is the second most intense ion, it was chosen as the lead daughter ion since it is common to both compounds. The correlation curve obtained for increasing concentrations of MTP and MTP-d7 evaluated by the area under the curve of the transitions from $268 \rightarrow 191.08$ for MTP and $275 \rightarrow 191.08$ for MTP-d7 had a coefficient of determination $R^{2}$ of 0.9999 (Supplemental Fig. 4C). Both MTP and MTP-d7 eluted at similar times (i.e., between 10 and 11 minutes; Supplemental Fig. 4D). Next, identical fragmentation patterns were found for both TAM and TAM$\mathrm{d} 5$, producing two daughter ions $(\mathrm{m} / z 228$ and $\mathrm{m} / z$ 271). To construct the correlation graph and achieve a coefficient of determination $R^{2}$ of 0.9998 (Supplemental Fig. 4G), the following transitions were followed: $409.00 \rightarrow 271$ for TAM and $414 \rightarrow 271$ for TAM-d5. The TAM and TAM-d5 elution profiles were indistinguishable. Both ions eluted between 10.2 and 10.8 minutes. DOX and DOX-d8 were identified by two ions: $m / z 452.25$ and $m / z 460.25$ (Supplemental Fig. 4 , I and J). The following transitions were monitored: $452.25 \rightarrow 344.25$ for DOX and $460.25 \rightarrow 352.25$ for DOX-d8. The correlation curve compiled for DOX and DOX-d8 resulted in a coefficient of determination $R^{2}$ of 0.9978 (Supplemental Fig. 4K). DOX and DOX-d8 shared identical elution patterns $(12.0 \rightarrow 12.5$ minutes $)$ under the specified chromatographic conditions. Finally, fragmentation of BRM and its chosen internal standard $\alpha$-ergocriptine resulted in two characteristic ions: $\mathrm{m} / z 656.7$ and $m / z$ 576.7, respectively. The following transitions were monitored: $\mathrm{m} / z \quad 656.70 \rightarrow 348.08$ for $\mathrm{BRM}$ and $\mathrm{m} / z$ $576.70 \rightarrow 268.17$ for $\alpha$-ergocriptine (Supplemental Fig. 4, M and $\mathrm{N}$ ). The correlation plot between BRM and $\alpha$-ergocriptine was found to have a coefficient of determination $R^{2}$ of 0.9923
(Supplemental Fig. 4O). Both BRM and $\alpha$-ergocriptine eluted between 11 and 12 minutes (Supplemental Fig. 4P). Several problems were encountered when attempting to design a deuterated derivative of BRM. Therefore, the best alternative was to use $\alpha$-ergocriptine. This standard satisfied almost all criteria except that its linearity differed from the linearity on the concentration interval when compared with BRM. Therefore, a corrective coefficient ( $c=29.5$ ) was used. This approach resolved the issue, and a test with known concentrations of BRM and $\alpha$-ergocriptine produced only a $4 \%-7 \%$ deviation from their real values. Deuterated standards for MTP, TAM, and DOX had correction coefficients with near ideal values of $1.05,1.1$, and 0.95 , respectively, over the concentration scale tested.

The absorption, retention, and elimination of the individual drugs and their various combinations (detailed in Fig. 5) were then determined in mouse sera and eyes. In almost all cases, drug levels in serum dropped to less than $5 \%$ of their maximum by 4 hours after treatment. In contrast, drug levels in the eyes remained unchanged over the 24-hour test period. Control experiments were performed that followed the main ion $\rightarrow$ daughter ion transitions in untreated samples to ensure that these transitions were present only in treated samples.

Drug levels in sera and eyes of treated mice were obtained at $1,2,4,8,12,16$, and 24 hours after treatment. For each drug, a minimal dose that showed an effect in preclinical studies and then higher doses were used for the analyses (Fig. 5). Serum levels dropped in 4 hours to less than $10 \%$ of those reached at 1 hour for single drugs. By 24 hours, drug levels were either undetectable or below $1 \%$ of the levels found 1 hour after treatment (Fig. 5, A, C, E, and G). Contrary to levels in sera, levels in the eyes for each drug at all doses maintained relatively constant levels (Fig. 5, B, D, F, and H). Treatments with multiple drugs were then conducted in a similar manner (Figs. 6 and 7). Combined treatments were administered either by intraperitoneal injection (Fig. 6) or gastric gavage (Fig. 7). Doses for these drugs (see Supplemental Table 2) were 

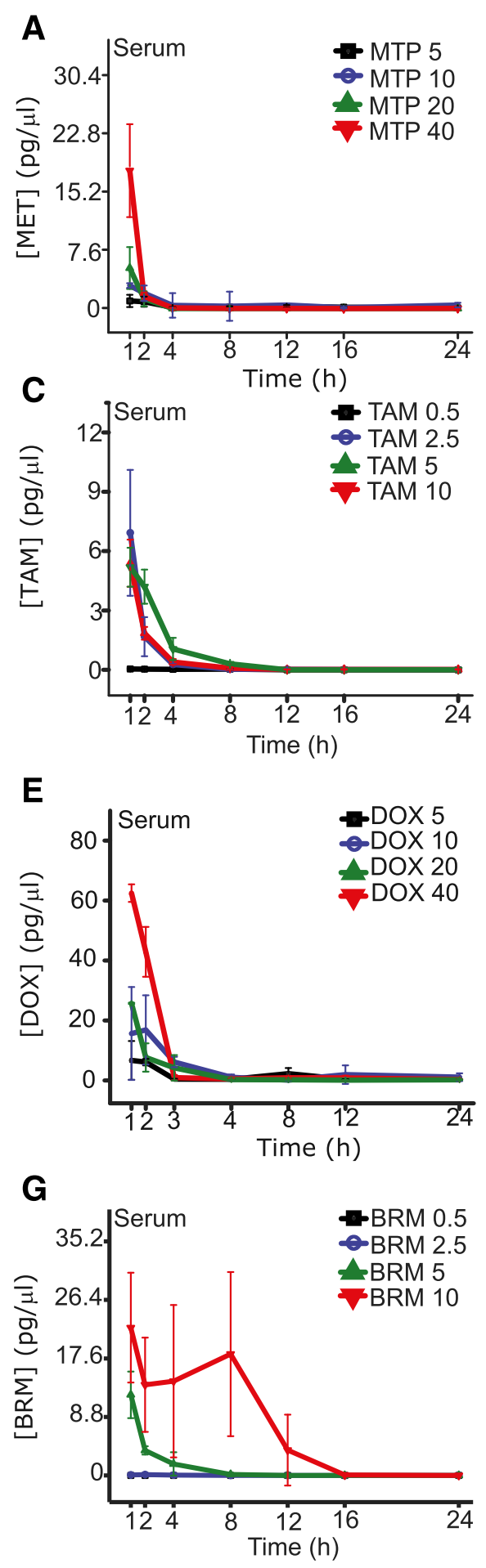
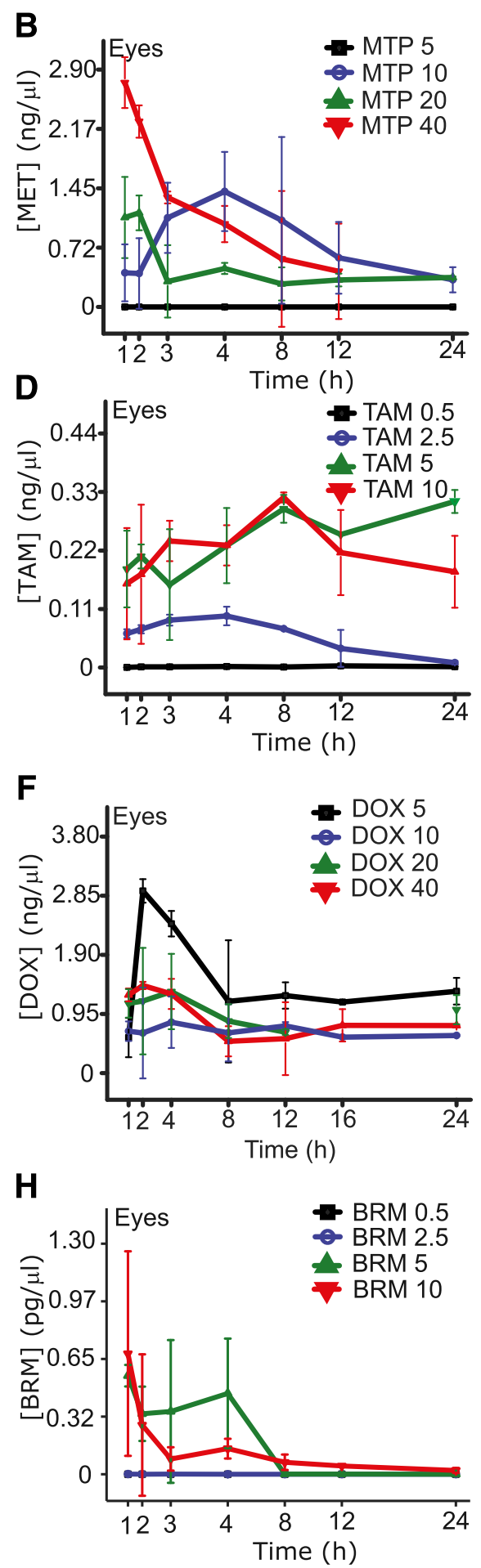

Fig. 5. Serum and eye levels of MTP, TAM, DOX, and BRM after single intraperitoneal injections. (A and B) Serum and eye levels of MTP. Levels corresponding to the 5, 10, 20, and $40 \mathrm{mg} / \mathrm{kg}$ doses are shown. (C and D) Serum and eye levels of TAM. Levels of the drug in the serum and eye corresponding to the $0.5,2.5,5$, and $10 \mathrm{mg} / \mathrm{kg}$ doses are shown. (E and F) Serum and eye levels of DOX. Levels of DOX corresponding to $5,10,20$, and $40 \mathrm{mg} / \mathrm{kg}$ doses are shown. ( $G$ and $H$ ) Serum and eye levels of BRM. Levels of BRM corresponding to 0.5, 2.5, 5, and $10 \mathrm{mg} / \mathrm{kg}$ doses are shown. evaluated and results obtained were similar to those from the single drug treatment studies. For the intraperitoneal injection protocol, a set of experiments was performed to determine drug levels in perfused and nonperfused samples of the eye (Fig. 6, C and F). Both methodologies gave similar results, suggesting only a small contribution from the blood in the eye sample.

To determine whether the blood-brain barrier (BBB) could have an effect on the amount of drug reaching the eye by opening the blood-retina barrier, MTP and DOX were selected as test agents. MTP can easily cross the BBB (Neil-Dwyer et al., 1981), whereas DOX is more restricted (Kamibayashi et al., 1995). One group of mice $(n=5)$ was treated for 4 days with fingolimod, a drug that permeabilizes the BBB (Yanagida et al., 2017), whereas a second group of mice received $0.9 \%$ $\mathrm{NaCl}$ for 4 days. On the fifth day, both groups were treated with MTP and DOX, each at doses of $1 \mathrm{mg} / \mathrm{kg}$ by intraperitoneal injection. DOX levels in the eyes were found to be lower in 

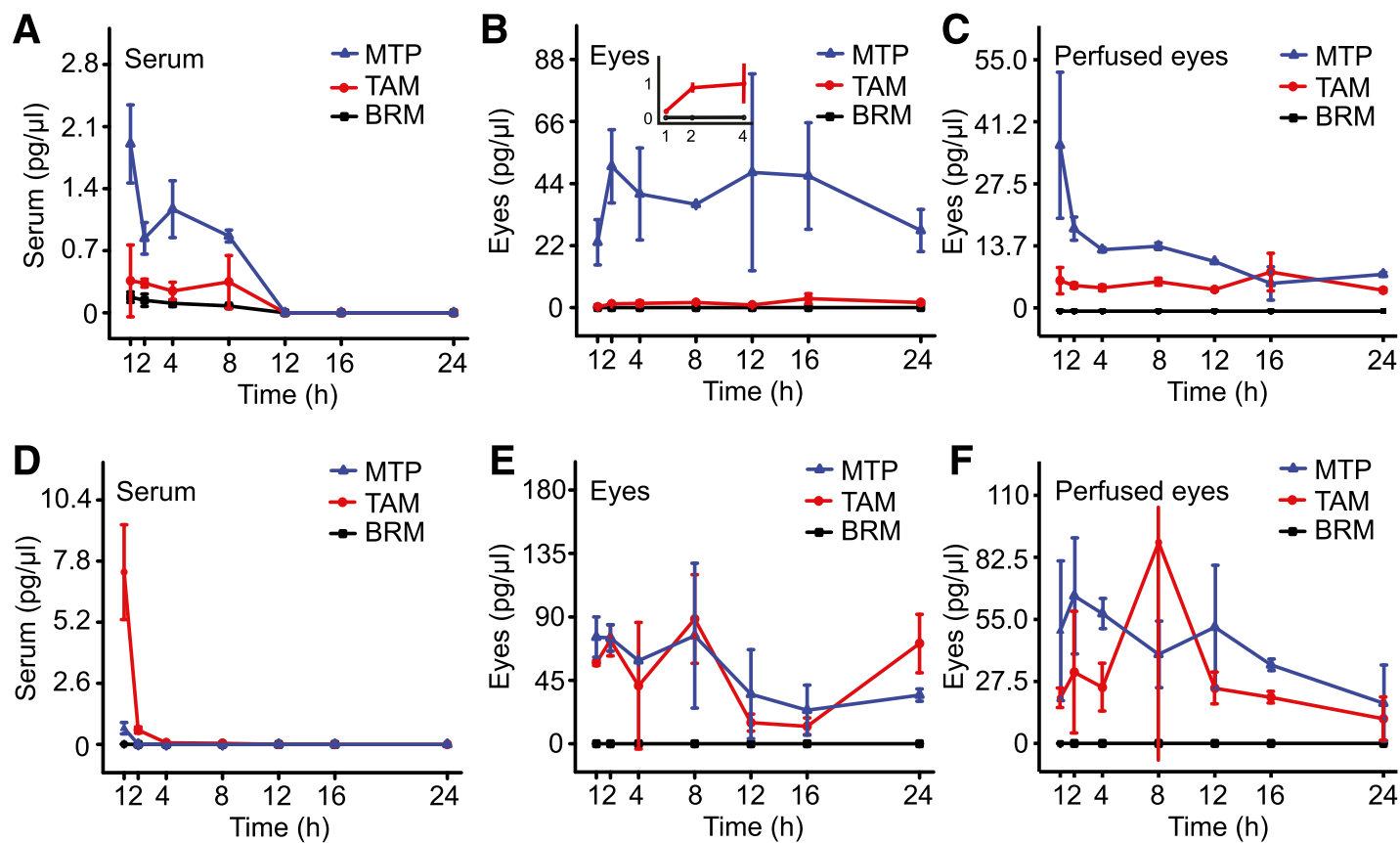

Fig. 6. Serum and eye levels of GPCR modulators given in combination by intraperitoneal injection. Drug levels are shown in serum and in nonperfused and perfused eye samples. (A) Serum levels after treatment with TAM + MTP + BRM; MTP, TAM, and BRM, were dosed at 1, 0.05, and 0.1 mg/kg, respectively. (B and C) Drug levels in the nonperfused and perfused eye. Doses used for treatment and color coding were identical to those in (A). (D) Serum levels after treatment with MTP + DOX + BRM. MTP (blue triangles), DOX (red circles), and BRM (black squares) were dosed at 1 , 1 , and $0.1 \mathrm{mg} / \mathrm{kg}$, respectively. (E and F) Drug levels in nonperfused and perfused eyes, respectively. Doses used for treatment and color coding were identical to those in (D).

the fingolimod-treated group. When levels of DOX were measured in the brains of the two groups, the level of DOX in the group treated with fingolimod was significantly higher than that in the control group (Fig. 8). This result indicates that fingolimod, as a BBB disruptor, can increase DOX levels in the brain but not the eye, suggesting that the blood-retina barrier is unaffected by fingolimod for tested drugs. As expected, no significant difference in MTP levels was found between the two groups, since MTP easily crosses the BBB without fingolimod. Statistical analyses of the pharmacokinetic data were performed using the $t$ test with the statistical significance level set at $P<0.05$.
A
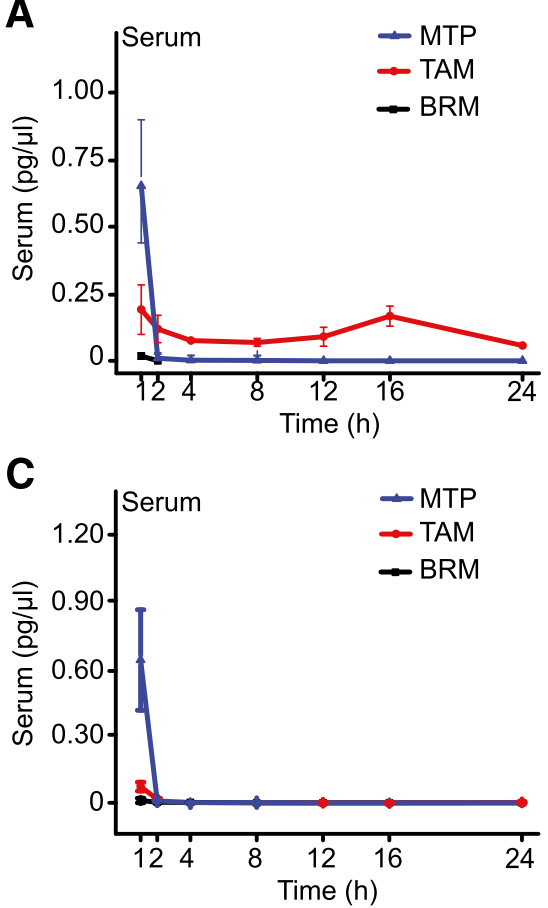
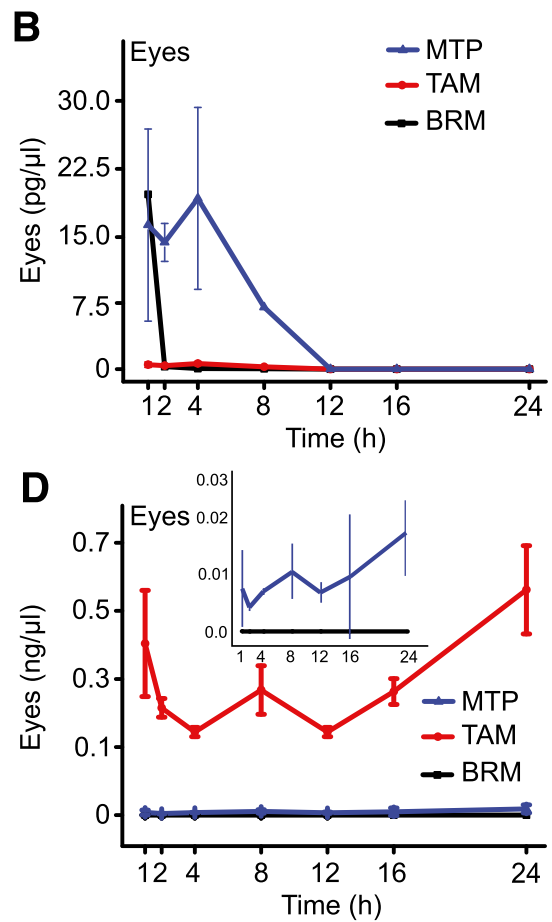

Fig. 7. Serum and eye levels of combinations of GPCR modulators administered by gastric gavage. Triple treatment by gastric gavage of nonperfused eye samples. (A) Drug levels in serum after treatment with MTP $+\mathrm{TAM}+\mathrm{BRM}$ administered at $0.05,2$, and $0.2 \mathrm{mg} / \mathrm{kg}$ doses, respectively. (B) Drug levels in the eye after treatment with MTP + TAM + BRM; doses are the same as in (A). (C) Serum levels after gastric gavage with MTP (blue triangles), DOX (red circles), and BRM (black squares) alone and with MTP + DOX + BRM administered at 2,2 , and $0.2, \mathrm{mg} / \mathrm{kg}$. (D) Drug levels in the nonperfused eye after treatment MTP + DOX + BRM; doses are the same as in (C) 

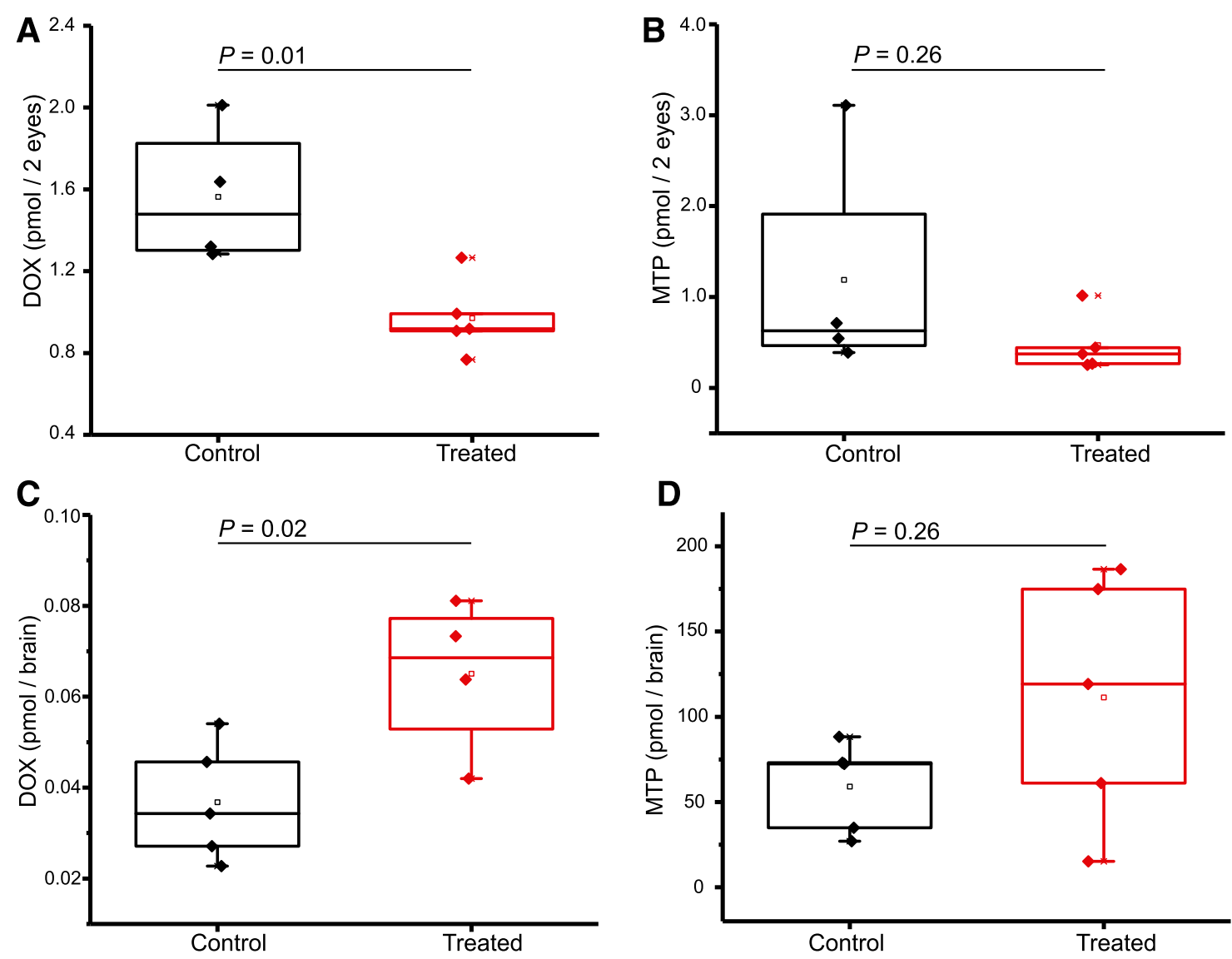

Fig. 8. BBB disruption in mice treated with DOX or MTP. (A and B) DOX and MTP levels in the eyes of mice treated by intraperitoneal injections of $1 \mathrm{mg} / \mathrm{kg}$. Samples labeled as controls were treated with $0.9 \% \mathrm{NaCl}$ for 4 days, followed by an intraperitoneal injection of a single dose of DOX or MTP. Samples labeled as treated were injected with fingolimod for 4 days at a dose set to $1 \mathrm{mg} / \mathrm{kg}$, followed by a single treatment with DOX or MTP. (C and D) The experimental design was identical to that described in (A) and (B) except that levels of DOX and MTP were measured in brain samples. Statistical significance was evaluated with a $t$ test. Differences between groups that resulted in $P$ values $<0.05$ were considered statistically significant.

\section{Discussion}

Our previous studies in mice demonstrated that the pathogenesis of light-induced retinopathy involves either increased signaling through $\mathrm{G}_{\mathrm{q}^{-}}$or $\mathrm{G}_{\mathrm{s}}$-coupled GPCRs or decreased activity of $\mathrm{G}_{\mathrm{i}}$-coupled GPCRs (Chen and Palczewski, 2015; Chen et al., 2016). Here, retinal protection was observed in $\mathrm{Abca4^{-/ }} R d h 8^{-1-}$ and BALB/cJ mice when MTP (a $\mathrm{G}_{\mathrm{s}}$-coupled pathway antagonist), TAM (a $\mathrm{G}_{\mathrm{q}}$ pathway antagonist), or BRM (a $\mathrm{G}_{\mathrm{i}}$ pathway agonist) was dosed individually at $1,2.5$, and $10 \mathrm{mg} / \mathrm{kg}$ (Chen et al., 2016). These initial studies focused almost exclusively on rod photoreceptors, whereas cone photoreceptor cell preservation is more relevant to daily human vision (Mustafi et al., 2009).

In this study, protection of cone cells was achieved by pharmacological intervention with a combination of the GPCR modulators MTP + TAM + BRM (Fig. 1). Both the structure and function of cone cells were preserved, as evidenced by different imaging analyses (Fig. 1, A, D, and E; Supplemental Fig. 2) and ERG (Fig. 1, B and C). However, this therapeutic effect was achieved over a short period, 0.5-2 hours prior to the onset of bright light. This observation agrees with the pharmacokinetic data (discussed below), showing that the majority of each GPCR modulator is cleared from the plasma within 4 hours after its intraperitoneal injection. Modes of drug administration other than bolus injection need to be investigated before further longitudinal studies are conducted in mice. The biological half-life of these drugs in serum is much longer in humans than in mice and rats (Jordö et al., 1979; Maurer et al., 1983; Lemmer et al., 1985; Matsushima et al., 1998), enabling once- or twice-daily administration for effective treatment of nonocular diseases in human patients. The enhanced half-life of these drugs in humans could bode well for use in treating retinal degenerative disorders.

In this study, the open-source PRESTO-TANGO assay was used for parallel and simultaneous interrogation of the "druggable" GPCR-ome for agonistic activity (Barnea et al., 2008). Results were obtained for four compounds (MTP, TAM, DOX, and BRM) tested against 315 GPCR targets. MTP, TAM, and DOX showed no agonistic activity, as expected. However, four potential ligand GPCR pairings were identified for BRM (namely, HTR1A, DRD2, DRD3, and ADRA2C). Moreover, dose-response curves generated for BRM included a doublesigmoidal curve suggesting a dual binding of BRM to DRD2. These data reveal a complex pharmacological and subsequent signaling profile for BRM.

Of further significance, GPCR targets identified by a systems pharmacology approach are normally expressed in human retina and conserved among species. Unfortunately, anti-GPCR antibodies are generally unreliable for cellular localization (Baker, 2015), so a PCR approach was chosen in 
addition to RNA-seq studies to evaluate GPCR expression in various species including humans (Table 1; Supplemental Table 4). Initially, qPCR was carried out to measure the expression of the 13 GPCRs shown to serve as targets for modulators capable of protecting photoreceptor cells in a bright light model of retinal degeneration (Chen et al., 2016). These GPCRs were found highly expressed (as shown by both qPCR and RNA-seq) in the retinas of humans and all tested mammals, suggesting their conserved functions. In addition to the 13 targeted GPCRs, most GPCRs were conserved, also indicating their functional importance. Furthermore, the experimental drugs did not significantly affect the expression of targeted GPCRs and other receptors present in the mouse eye. Obviously, expression levels do not necessarily reflect actual receptor levels, as the latter are influenced by the stability of the corresponding mRNA and the translated product.

The systems pharmacology approach to examine the impact of mono- and combined drug pretreatments in mouse models of bright light-induced retinal degeneration engaged a global transcriptome strategy using RNA-seq (Chen et al., 2016) and methods previously developed (Mustafi et al., 2013b). The findings demonstrated that expression of opsin photopigments declined upon exposure to bright light, correlating with the loss of photoreceptor cells. This effect on opsin expression was mitigated by drug treatment, as shown by RNA-seq analysis, which again correlated with the protection of photoreceptor cells exposed to bright light. When used in combination, the dose of individual drugs could be reduced 10-fold compared with their individual effective dose and still maintain their ability to rescue opsin expression (Fig. 3). Similar effect has been shown earlier in preservation of photoreceptor structure and function (Chen et al., 2016). (Chen et al., 2016). This observation highlights the cooperative action of these drug combinations in vivo. Collectively, these results also demonstrate the preservation of photoreceptor cells that comprise the main sites of opsin expression.

Although combinations of Food and Drug Administrationapproved drugs might prevent light-induced retinal damage in mouse models, they also could adversely affect normal retinal function, thereby limiting their clinical use. ERG responses reflect the electrical activities of various cell types in the retina including rod and cone photoreceptors. Here, ERG recordings were essentially the same in terms of both their a-wave and b-wave amplitudes among the three experimental groups, two combination drug therapy groups and one DMSO control group. These findings satisfy at least one significant precondition for the use of these drugs to treat retinal degenerative diseases.

Prior to clinical application of a therapy, however, pharmacokinetic data are essential for the safe and effective management of drugs in patients. Therefore, applying our developments in sample processing, internal standards, and LC-MS methods, we undertook initial pharmacokinetic studies of GPCR modulators capable of protecting photoreceptor cells in our mouse model of retinal degeneration. LC-MS methods developed here allowed the identification and quantification of MTP, TAM, DOX, and BRM in a manner that produced significant correlations between each drug and its corresponding internal standard, with $R^{2}$ values $>0.99$ Supplemental Fig. 4, C, G, K, and O). This increased the accuracy of evaluating drug levels in different tissues with minimal sample processing. In addition to improvements in LC-MS methods, perfusion experiments were performed to rule out sample contamination by blood during processing. Nonperfused eyes were found to contain similar levels of each drug as in their perfused counterparts (Fig. 6, B and E and C and F, respectively). This result demonstrates that these drugs persisted in the eye and were not easily diluted by the circulation or the perfusion solution (Fig. 6, C and F). Two different modes of administration, intraperitoneal and gastric gavage, also were tested. The oral dose used here was greater than that of the same treatment given intraperitoneally to compensate for lower absorption from the gastrointestinal tract. Serum levels of drugs administered by gastric gavage were lower than those found after intraperitoneal administration (Figs. 6 and 7). In contrast, drug levels in the eyes, especially for DOX, were found to be considerably higher after oral administration and were sufficient to protect photoreceptor cells in the light-induced mouse model of retinal degeneration with no adverse structural or functional effects. Along with the longer biologic half-life of GPCR modulators in humans, oral administration can provide a safe and effective route for combination drug therapy.

An avenue worth exploring prior to clinical trials is to formulate MTP + TAM + BRM as eye drops and test their efficacy experimentally. A topical route of administration would further minimize adverse effects. Successful therapeutic outcomes after topical administration of adrenergic drugs for ocular conditions already exist. Timolol (a $\beta$-blocker) and brimonidine (a $\alpha-2$ receptor agonist) are clinically used as eye drops to treat ocular hypertension and glaucoma. Moreover, brimonidine eye drops have shown protective effects against photoreceptor death in a focal light damage model in mice (Ortín-Martínez et al., 2014).

When administered after selective opening of the BBB with fingolimod, MTP levels in the eye were unaffected. This result is not surprising, since MTP easily crosses the BBB. In contrast, fingolimod enhanced the levels of DOX in the brain but had no demonstrable effect on drug levels in the eye. Overall, pharmacokinetic studies demonstrate that the protective effects of combination therapy with GPCR modulators correlate better with serum concentrations of the drugs than their levels in the eye. This protective effect could be due to partitioning of these drugs into eye compartments irrelevant to their therapeutic effect (i.e., the vitreous). Therefore, more detailed distributional studies are needed to fully appreciate the pharmacokinetic data.

In summary, a systems pharmacology approach has now identified a combination of GPCR modulators, which effectively protect photoreceptor cells in mouse models of retinal degeneration without deleterious effects on the processing of light signals through the retina. Levels of these modulatory drugs sufficient to protect photoreceptor cells were found in the eye after both intraperitoneal and oral administration, and pharmacokinetic data tended to support their prolonged elimination from the eye relative to plasma. Although additional studies are warranted, these data support the transition of a systems pharmacology-based therapy consisting of GPCR drugs such as MTP, DOX, TAM, and BRM into clinical evaluation.

\section{Acknowledgments}

We thank Dr. Leslie T. Webster Jr. and members of the K.P. and P.D.K. laboratories for their helpful comments regarding this 
manuscript. We also thank members of the National Institutes of Health National Institute of Mental Health (NIMH) Psychoactive Drug Screening Program (PDSP; contract HHSN-271-2013-00017-C) for conducting the GPCR-ome screen. The NIMH Psychoactive Drug Screening Program is directed by Bryan L. Roth (University of North Carolina, Chapel Hill, NC) and Project Officer Jamie Driscoll (NIMH, Bethesda, MD). We thank Dr. Yu Chen (Shanghai University, Shanghai, China) and Dr. Debarshi Mustafi (University of Southern California, Los Angeles, CA) for generating the RNA-seq data, Anthony Gardella (CWRU, Visual Sciences Research Core) for technical assistance in the analysis of retinal images, and Xiuli Ma for help with the perfusion experiments.

\section{Authorship Contributions}

Participated in research design: Orban, Leinonen, Kiser, Palczewski.

Conducted experiments: Orban, Leinonen, Getter, Dong, Sun, Gao, Veenstra, Heidari-Torkabadi.

Performed data analysis: Orban, Leinonen, Getter, Gao, Palczewski.

Wrote or contributed to the writing of the manuscript: Orban, Leinonen, Kern, Kiser, Palczewski.

\section{References}

Anderson DH and Jacobs GH (1972) Color vision and visual sensitivity in the California ground squirrel (Citellus beecheyi). Vision Res 12:1995-2004.

Baker M (2015) Reproducibility crisis: blame it on the antibodies. Nature 521: $274-276$.

Barnea G, Strapps W, Herrada G, Berman Y, Ong J, Kloss B, Axel R, and Lee KJ (2008) The genetic design of signaling cascades to record receptor activation. Proc Natl Acad Sci USA 105:64-69.

Bessant DA, Payne AM, Mitton KP, Wang QL, Swain PK, Plant C, Bird AC, Zack DJ, Swaroop A, and Bhattacharya SS (1999) A mutation in NRL is associated with autosomal dominant retinitis pigmentosa. Nat Genet 21:355-356.

Chen Y, Okano K, Maeda T, Chauhan V, Golczak M, Maeda A, and Palczewski K (2012) Mechanism of all-trans-retinal toxicity with implications for Stargardt disease and age-related macular degeneration. J Biol Chem 287:5059-5069.

Chen Y, Palczewska G, Masuho I, Gao S, Jin H, Dong Z, Gieser L, Brooks MJ, Kiser PD, Kern TS, et al. (2016) Synergistically acting agonists and antagonists of G protein-coupled receptors prevent photoreceptor cell degeneration. Sci Signal $\mathbf{9}$ ra74.

Chen Y, Palczewska G, Mustafi D, Golczak M, Dong Z, Sawada O, Maeda T, Maeda A, and Palczewski K (2013) Systems pharmacology identifies drug targets for Stargardt disease-associated retinal degeneration. J Clin Invest 123:5119-5134

Chen Y and Palczewski K (2015) Systems pharmacology links GPCRs with retinal degenerative disorders. Annu Rev Pharmacol Toxicol 56:273-298

Curcio CA, Medeiros NE, and Millican CL (1996) Photoreceptor loss in age-related macular degeneration. Invest Ophthalmol Vis Sci 37:1236-1249.

Curcio CA, Sloan KR, Kalina RE, and Hendrickson AE (1990) Human photoreceptor topography. J Comp Neurol 292:497-523.

Humphries MM, Rancourt D, Farrar GJ, Kenna P, Hazel M, Bush RA, Sieving PA Sheils DM, McNally N, Creighton P, et al. (1997) Retinopathy induced in mice by targeted disruption of the rhodopsin gene. Nat Genet 15:216-219.

Jeon CJ, Strettoi E, and Masland RH (1998) The major cell populations of the mouse retina. $J$ Neurosci 18:8936-8946.

Jordö L, Johnsson G, Lundborg P, Persson BA, Regärdh CG, and Rönn O (1979) Bioavailability and disposition of metoprolol and hydrochlorothiazide combined in one tablet and of separate doses of hydrochlorothiazide. Br J Clin Pharmacol 7 : 563-567.

Kamibayashi T, Hayashi Y, Mammoto T, Yamatodani A, Sumikawa K, and Yoshiya I (1995) Role of the vagus nerve in the antidysrhythmic effect of dexmedetomidine on halothane/epinephrine dysrhythmias in dogs. Anesthesiology 83:992-999.

Katritch V, Cherezov V, and Stevens RC (2013) Structure-function of the G proteincoupled receptor superfamily. Annu Rev Pharmacol Toxicol 53:531-556.
Kroeze WK, Sassano MF, Huang XP, Lansu K, McCorvy JD, Giguère PM, Sciaky N, and Roth BL (2015) PRESTO-Tango as an open-source resource for interrogation of the druggable human GPCRome. Nat Struct Mol Biol 22:362-369.

Lem J, Krasnoperova NV, Calvert PD, Kosaras B, Cameron DA, Nicolò M, Makino CL, and Sidman RL (1999) Morphological, physiological, and biochemical changes in rhodopsin knockout mice. Proc Natl Acad Sci USA 96:736-741.

Lemmer B, Winkler H, Ohm T, and Fink M (1985) Chronopharmacokinetics of betareceptor blocking drugs of different lipophilicity (propranolol, metoprolol, sotalol, atenolol) in plasma and tissues after single and multiple dosing in the rat. Naunyn Schmiedebergs Arch Pharmacol 330:42-49.

Maeda A, Maeda T, Golczak M, Chou S, Desai A, Hoppel CL, Matsuyama S, and Palczewski K (2009) Involvement of all-trans-retinal in acute light-induced retinopathy of mice. J Biol Chem 284:15173-15183.

Maeda A, Maeda T, Golczak M, and Palczewski K (2008) Retinopathy in mice induced by disrupted all-trans-retinal clearance. J Biol Chem 283:26684-26693.

Maj J, Gancarczyk L, and Rawlów A (1977) The influence of bromocriptine on serotonin neurons. J Neural Transm (Vienna) 41:253-264.

Matsushima H, Kamimura H, Soeishi Y, Watanabe T, Higuchi S, and Tsunoo M (1998) Pharmacokinetics and plasma protein binding of tamsulosin hydrochloride in rats, dogs, and humans. Drug Metab Dispos 26:240-245.

Maurer G, Schreier E, Delaborde S, Nufer R, and Shukla AP (1983) Fate and disposition of bromocriptine in animals and man. II: absorption, elimination and metabolism. Eur J Drug Metab Pharmacokinet 8:51-62.

McBee JK, Palczewski K, Baehr W, and Pepperberg DR (2001) Confronting complexity: the interlink of phototransduction and retinoid metabolism in the vertebrate retina. Prog Retin Eye Res 20:469-529.

Mears AJ, Kondo M, Swain PK, Takada Y, Bush RA, Saunders TL, Sieving PA, and Swaroop A (2001) Nrl is required for rod photoreceptor development. Nat Genet 29:447-452.

Mustafi D, Engel AH, and Palczewski K (2009) Structure of cone photoreceptors. Prog Retin Eye Res 28:289-302.

Mustafi D, Kevany BM, Bai X, Golczak M, Adams MD, Wynshaw-Boris A, and Palczewski K (2016) Transcriptome analysis reveals rod/cone photoreceptor specific signatures across mammalian retinas. Hum Mol Genet 25:4376-4388.

Mustafi D, Kevany BM, Bai X, Maeda T, Sears JE, Khalil AM, and Palczewski K (2013a) Evolutionarily conserved long intergenic non-coding RNAs in the eye. Hum Mol Genet 22:2992-3002.

Mustafi D, Kevany BM, Genoud C, Bai X, and Palczewski K (2013b) Photoreceptor phagocytosis is mediated by phosphoinositide signaling. FASEB J 27:4585-4595.

Mustafi D, Kevany BM, Genoud C, Okano K, Cideciyan AV, Sumaroka A, Roman AJ, Jacobson SG, Engel A, Adams MD, et al. (2011) Defective photoreceptor phagocytosis in a mouse model of enhanced S-cone syndrome causes progressive retinal degeneration. FASEB $J$ 25:3157-3176.

Neil-Dwyer G, Bartlett J, McAinsh J, and Cruickshank JM (1981) Beta-adrenoceptor blockers and the blood-brain barrier. Br J Clin Pharmacol 11:549-553.

Nikonov SS, Kholodenko R, Lem J, and Pugh EN, Jr (2006) Physiological features of the S- and M-cone photoreceptors of wild-type mice from single-cell recordings. $J$ Gen Physiol 127:359-374.

Ortín-Martínez A, Valiente-Soriano FJ, García-Ayuso D, Alarcón-Martínez L, Jiménez-López M, Bernal-Garro JM, Nieto-López L, Nadal-Nicolás FM, Villegas-Pérez MP, Wheeler LA, et al. (2014) A novel in vivo model of focal light emitting diodeinduced cone-photoreceptor phototoxicity: neuroprotection afforded by brimonidine, BDNF, PEDF or bFGF. PLoS One 9:e113798.

Rosenfeld MR, Makman MH, Ahn HS, Thal LJ, Mishra RK, and Katzman R (1980) Selective influence of ergot alkaloids on cortical and striatal dopaminergic and sergotonergic receptors. Adv Biochem Psychopharmacol 23:83-93.

Szél A, von Schantz M, Röhlich P, Farber DB, and van Veen T (1993) Difference in PNA label intensity between short- and middle-wavelength sensitive cones in the ground squirrel retina. Invest Ophthalmol Vis Sci 34:3641-3645.

Wenzel A, Grimm C, Samardzija M, and Reme CE (2005) Molecular mechanisms of light-induced photoreceptor apoptosis and neuroprotection for retinal degeneration. Prog Retin Eye Res 24:275-306.

Yanagida K, Liu CH, Faraco G, Galvani S, Smith HK, Burg N, Anrather J, Sanchez T, Iadecola C, and Hla T (2017) Size-selective opening of the blood-brain barrier by targeting endothelial sphingosine 1-phosphate receptor 1. Proc Natl Acad Sci USA 114:4531-4536.

Address correspondence to: Krzysztof Palczewski, Department of Pharmacology, School of Medicine, Case Western Reserve University, 10900 Euclid Ave., Cleveland, OH 44106. E-mail: kxp65@case.edu 